\title{
Reconfigurable Intelligent Surfaces Aided Multi-Cell NOMA Networks: A Stochastic Geometry Model
}

\author{
Chao Zhang, Student Member, IEEE, Wenqiang Yi, Student Member, IEEE, Yuanwei Liu, Senior Member, IEEE, \\ Kun Yang, Senior Member, IEEE, and Zhiguo Ding, Fellow, IEEE
}

\begin{abstract}
By activating blocked users and altering successive interference cancellation (SIC) sequences, reconfigurable intelligent surfaces (RISs) become promising for enhancing nonorthogonal multiple access (NOMA) systems. To evaluate the benefits between RISs and NOMA, a downlink RIS-aided multicell-NOMA network is investigated via stochastic geometry. We first introduce the unique path loss model for RIS reflecting channels. Then, we evaluate the angle distributions based on a Poisson cluster process (PCP) model, which theoretically demonstrates that the angles of incidence and reflection are uniformly distributed. Additionally, we derive closed-form analytical and asymptotic expressions for coverage probabilities of the paired NOMA users. Lastly, we derive the analytical expressions of the ergodic rate for both of the paired NOMA users and calculate the asymptotic expressions for the typical user. The analytical results indicate that 1) the achievable rates reach an upper limit when the length of RIS increases; 2) exploiting RISs can enhance the path loss intercept to improve the performance without influencing the bandwidth. The simulation results show that 1) RIS-aided networks have superior performance than the networks without RISs; and 2) the SIC order in NOMA systems can be altered since RISs are able to change the channel quality of NOMA users.
\end{abstract}

Index Terms-Multi-cell NOMA, reconfigurable intelligent surface, stochastic geometry

\section{INTRODUCTION}

Due to introducing new freedom, non-orthogonal multiple access (NOMA) evolves into a promising technique. By sharing spectrum with power multiplexing schemes and successive interference cancellation (SIC), the spectral efficiency and user connectivity enable to be significantly improved to satisfy different target requirements [2]. In spite of benefits, NOMA techniques still have several significant implementation challenges such as lower received power and severer interference than orthogonal multiple access (OMA) users. To cope with the challenges, reconfigurable intelligent surfaces (RIS), also known as intelligent reflecting surfaces (IRS), are promising in several aspects $[3,4]$. On the one hand, environmental

C. Zhang, W. Yi, and Y. Liu are with the School of Electronic Engineering and Computer Science, Queen Mary University of London, London, UK (email:\{chao.zhang, w.yi, yuanwei.liu\}@qmul.ac.uk).

$\mathrm{K}$. Yang is with the School of Computer Science and Electronic Engineering, The University of Essex, Colchester, U.K. (email: kunyang@essex.ac.uk).

Z. Ding is with the School of Electrical and Electronic Engineering, The University of Manchester, Manchester, UK (e-mail: zhiguo.ding@manchester.ac.uk).

Part of this work has been accepted to be presented in IEEE Global Communications Conference (GLOBECOM), Taipei, China, December, 2020 $[1]$. obstacles may ruin the required channel condition, especially for the far NOMA users blocked by high buildings, which results in inevitable outage situations. With the aid of RISintroduced line-of-sight (LoS) propagation, we are able to exploit reflecting links through RISs to improve the channel condition of blocked NOMA users [5]. On the other hand, RISs are enabled to achieve flexible decoding orders according to the quality of service (QoS) conditions.

A RIS is regarded as a two-dimensional-equivalent reconfigurable meta-material, which consists of elementary elements called scattering particles or meta-atoms [6,7]. Based on intelligent meta-surface technologies, RISs have properties such as absorbing incident waves or modifying the reflected wavefronts $[8,9]$. In contrast to mirrors, RISs are able to adjust the angle of reflection and electric field strength. A major and basic open research challenge is to investigate the path loss model of RIS reflecting channels. Recent research contributions have studied the path loss model based on two typical methods, which are 1) correlated to the sum of incidence and reflection distances, and 2) correlated to the product of these distances. According to a fundamental work [7], both of the typical methods are correct but utilizing in different application scenarios such that: 1) "sum of distances" model is suitable for short-distance communications such as indoor scenarios, while 2) "product of distances" model is suitable for long-distance communications such as outdoor scenarios. To reduce the path loss and interference, RISs are placed near to the served NOMA user. This spatial grouping property is depicted by a tractable stochastic geometry model, namely the Poisson cluster process (PCP) [10-12], which provides a theoretical framework for investigating the average performance of RIS-aided NOMA networks.

\section{A. Related Works}

1) Related Works for RIS: Sparked by the aforementioned potential benefits of RISs, recent research contributions on RIS-aided networks have been evaluated in various aspects. Firstly, one promising topic is to propose the passive beamforming designs, i.e., passive beamforming and information transfer techniques with a sample average approximation based iterative algorithm and a turbo message passing algorithm [13], passive beamforming with modulation and resource allocation [14], and the achievable rates where limited discrete phase shifts are realized by the RIS [15]. Additionally, with 
various optimization designs, the performance of the RISaided networks is significantly enhanced: 1) the reflecting and precoding designs to minimize the symbol error rate were evaluated [16]; 2) the weighted sum rate by jointly designing the beamforming and the phase vector of the RIS was maximized [17]; 3) the asymptotic results of the maximum and minimum signal-to-interference-plus-noise-ratio (SINR) was investigated [18]. Moreover, contributions on deep learning methods have been investigated via $[19,20]$ and other related works with the aid of RIS have been investigated in several scenarios, such as mmWave environments [21,22], Internet of Things networks [23], and RIS-empowered multi-input-multi-output (MIMO) systems [24].

2) Related Works for RIS-aided NOMA: Additionally, with the aid of the RIS, the amounts of applications on RIS-NOMA systems attract our attention [25-29]. More specifically, an uplink RIS-aided NOMA system has been investigated to maximize the sum-rate [25]. Analytical results of the bit error rate (BER) for the downlink RIS-aided NOMA systems have been analyzed [26]. Moreover, the optimization on powerefficient for RIS-aided NOMA systems has been considered in [27-29], i.e., optimizing the beamforming vectors and the IRS phase shift matrix compared with zero-forcing beamforming [27], comparing power efficient of NOMA and OMA cases with the aid RISs [28], and proposing a difference-of-convex algorithm and efficient user ordering scheme to minimize the power consumption [29]. With respect to physical layer analysis on RIS-NOMA networks, several contributions have provided with the approaches to investigate the RIS-aided NOMA networks, such as several RIS-NOMA beamforming methods in [30] and deriving outage probability of a single-cell RIS-NOMA network in [31]. With the aid of former efforts, contributions on RIS-aided NOMA networks have been driven [32-34]. In detail, the outage probability and the ergodic rate for both downlink and uplink scenarios under the RISaided single-cell NOMA networks have been derived [33]. Additionally, the stochastic geometry models to evaluate the spacial effect under RIS-aided single-cell NOMA networks have been considered in $[32,34]$.

3) Related Works for RIS-aided multi-cell networks: Moreover, RIS-aided multi-cell networks are evaluated in several aspects [35-39]. First, a joint association optimization method is proposed for the downlink network with multiple base stations (BSs), users, and RISs to achieve the best performance with the BS-user association in conventional wireless networks without RISs as the benchmark [35]. A joint transmit and reflective beamforming for a RIS-aided multi-cell multipleinput single-output (MISO) system is designed [36]. To avoid deploying more BSs with high cost and power consumption, RIS-aided cell-free networks are proposed to improve the network capacity with low cost and power consumption [37]. Additionally, the cell boundary of multiple cells is investigated to assist the downlink transmission to cell-edge users with the aid of RISs [38]. For NOMA networks, a multi-cell RISaided NOMA network is considered to optimize the power allocation [39]. Although some RIS-aided multi-cell networks have been evaluated, the impact in terms of the randomness of networks is still in its infancy. Additionally, as most of works exploit a fixed number of cells to optimize the performance, one question is how to extend the multi-cell scenarios into an infinite area.

\section{B. Motivation and Contributions}

With the aid of stochastic geometry methods to capture the spatial effect of users and BSs, we extend the RISaided NOMA networks from single-cell scenarios to multicell scenarios to investigate the performance of practical cases. Hence, we present our motivations as follows:

- For RIS-aided multi-cell networks, most of the current papers are focusing on optimization algorithms, while the physical layer performance analysis is still a challenging task. Additionally, for performance analysis, tractable channel models, i.e., path loss models and fading effect, are needed for RIS-aided multi-cell networks, while this is still in their infancy.

- This paper aims to evaluate the spatial effect of the RISaided multi-cell-NOMA system with the aid of stochastic geometry models. We introduce a general path loss model, followed by an application of the "product of distances" model to analyze multi-cell NOMA networks for outdoor scenarios via a PCP-based spatial model.

- Since RISs are capable to emit signals from BSs to various directions, we motivate to exploit RISs to enhance the channel quality of blocked users.

- Since the quality of RIS-aided channels is better than the non-RIS-aided channels, we are able to alter the SIC order. Additionally, for QoS-based SIC ${ }^{1}$, RISs avoid delay-sensitive near users to accomplish SIC processes and enhance the SIC success rate of far delay-tolerant users.

Motivated by the aforementioned challenges, we investigate RIS-aided multi-cell NOMA networks. The main contributions are summarized as follows:

- We express a general model of RIS reflecting links. Based on the general case of RIS reflecting links, we derive the path loss model in long-distance regions. By modeling the multi-cell networks as a PCP distribution, we investigate the angle distributions, which verify that the angles formed by users, RISs and BSs are uniformly distributed in $[0, \pi]$.

- We derive closed-form analytical expressions for coverage probabilities of the paired NOMA users to enhance the evaluation efficiency. To investigate the impact of RISs, we calculate the asymptotic expressions for the typical user versus the half-length of RISs $L$. The analytical results indicate that we are capable of improving the coverage probability by adding the length of RISs.

- We derive the closed-form analytical expressions for ergodic rates of the paired NOMA users. Additionally, we calculate the asymptotic ergodic rate expressions for the typical user versus the half-length of RISs. The analytical results show that the achievable rates reach an upper

\footnotetext{
${ }^{1}$ QoS-based SIC is introduced in [40] with delay-sensitive user and delaytolerant users.
} 
limit when continuously enhancing the length of RISs. Additionally, enlarging the length of RISs can improve the path loss intercept, thereby enhance the coverage performance.

- Numerical results illustrate the following conclusions. 1) For the connected user, RIS-aided NOMA channels have superior coverage performance than RIS-aided OMA channels while it is on the contrary for the typical user. Additionally, for both NOMA users, RIS-aided networks acquire significantly enhanced performance than conventional scenarios without RISs. 2) If near users are delaysensitive users, we can exploit the RISs to avoid the SIC procedures at near users. This is because the RISs have the ability to enhance the channel quality effectively to alter the SIC orders.

\section{Organizations}

The remaining sections of this paper are organized as follows. In Section II, the system model of this RIS-aided multicell NOMA networks is introduced, including the path loss model of RIS reflecting links and signal models. In Section III, we derive the path loss models for the long-distance communication scenarios, including the analysis of the angle and distance distributions. In Section IV, we derive the closedform analytical expressions of coverage probability for the paired NOMA users. Additionally, we derive the asymptotic expressions of coverage probability for the typical user versus the length of RIS to evaluate the impact of RIS. In section $\mathrm{V}$, we investigate the ergodic rate performance with closedform analytical and asymptotic expressions. Numerical results are indicated in Section VI, followed by the conclusions in Section VII.

\section{System ModeL}

This paper considers RIS-aided downlink NOMA networks, where BSs and users are modeled according to two independent homogeneous Poisson point processes (HPPPs), namely $\Phi_{b} \subset \mathbb{R}^{2}$ with density $\lambda_{b}$ and $\Phi_{u} \subset \mathbb{R}^{2}$ with density $\lambda_{u}$, respectively. We consider RISs performing as linear materials, a sheet of electromagnetic material of negligible thickness. Additionally, we assume that two-user NOMA groups are served by orthogonal frequencies to cancel intra-cell interference. In each group, we assume that one of the paired users has already been connected to a BS in the previous user association process [10]. The other one, namely the typical user ${ }^{2}$, joins this occupied resource block by applying power-domain NOMA techniques. To simplify the analysis, the connected user is not included in the user set $\Phi_{u}$ and the distance between this user to its $\mathrm{BS}$ is invariable as $r_{c}$. The typical user is randomly selected from $\Phi_{u}$ and its location is fixed at the origin $\mathbb{O}(0,0)$

${ }^{3}$ of the considered plane, namely $\mathbf{x}_{U}$.

\footnotetext{
${ }^{2}$ We assume that the typical user is deployed in a cell with dense obstacles, i.e., tall buildings, thus the links from BSs to the user are blocked severely [12]. We additionally consider the RISs are deployed on tall buildings, hence the deployment of RISs is random.

${ }^{3}$ For BSs located in the RIS ball area $\mathbb{O}\left(0, R_{L}\right)$, RISs may weaken their direct LoS transmission due to phase difference [4]. Coherent transmission is desired for this case, which is beyond the scope of this paper.
}

\section{A. LoS Ball Model}

The blockage model is significant for RIS-aided networks as one promising application of RISs is to enhance the performance of blocked users by providing LoS transmission [4]. We consider an LoS ball model for the typical user in this work $[12,41]$. For the typical user, its LoS ball has a radius $R_{L}$. The transmitters within this ball provide LoS transmission, while those outside this ball have NLoS links. The LoS ball region of the typical user is given by $\mathbb{O}\left(0, R_{L}\right) \subset \mathbb{R}^{2}$, where $\mathbb{O}(a, b)$ represents an annulus with the inner radius $a$ and outer radius $b$. To ensure the RIS-to-User (RU) link is LoS, the RIS is uniformly distributed in the LoS ball area of the typical user. Due to considering a blocked typical user, the region of considered BSs is in the range $\mathbb{O}\left(R_{L}, \infty\right)$.

\section{B. RIS-aided Link Model}

We assume one RIS is employed for helping the typical user $^{4}$. Based on stochastic geometry principles and the randomness of the typical user, users and their serving RISs can be regarded as the Matern cluster process (MCP) pattern of PCP models with a fixed number of nodes in each cluster. More specifically, the possible typical users are the parent point process deployed by HPPPs, where we choose one of them as the considered typical user. The RISs are uniformly deployed in the clusters (LoS balls) as the daughter point process. The channel conditions of the connected user have been known with a fixed distance. Based on the MCP model, there are three significant communication links in the considered NOMA group: 1) The BS-to-User (BU) link, the link between the typical user and its BS; 2) The BS-to-RIS (BR) link, the link between the BS and the employed RIS; and 3) The RU link, the link between the RIS and the typical user. This work focuses on analyzing a blocked typical user and the RIS is applied to establish LoS route between the typical user and BSs [4]. Therefore, the BU link is assumed to be NLoS and the BR and RU links are LoS. Moreover, all NLoS communications are ignored in this paper due to their negligible received power.

The association criterion for the typical user is to associate with the BS offering the highest received power, which means that the distance between the RIS and the associated BS is the nearest. We assume the locations of the RIS and the associated BS are $\mathbf{x}_{R}$ and $\mathbf{x}_{B}$. Therefore, the distance between the associated BS and the RIS is correspondingly expressed as

$$
\mathbf{x}_{B R}=\arg \min _{\mathbf{x}_{B} \in \Phi_{B}}\left\|\mathbf{x}_{B}-\mathbf{x}_{R}\right\|,
$$

where $\mathbf{x}_{R} \in \mathbb{O}\left(0, R_{L}\right), \Phi_{B} \subset \Phi_{b}, \Phi_{B} \subset \mathbb{O}\left(R_{L}, \infty\right)$ and the location of arbitrary interfering BS is denoted by $\mathbf{x}_{I} \in \Phi_{r} \backslash \mathbf{x}_{B}$.

\section{Path Loss Model}

This work considers a $2 L$ linear RIS, whose central point is located at $\mathbf{X}_{R(0)}=\left(x_{R(0)}, y_{R(0)}\right) \subset \mathbb{O}\left(0, R_{L}\right)$. We define the

\footnotetext{
${ }^{4}$ As RISs have a significant influence on enhancing the performance of users, we consider one RIS is enough for the typical user on the performance enhancement. Hence, we can assume a few RISs are deployed in large areas, thus no more RIS in the same LoS ball. The cases with densely deployed RISs are left for future works.
} 


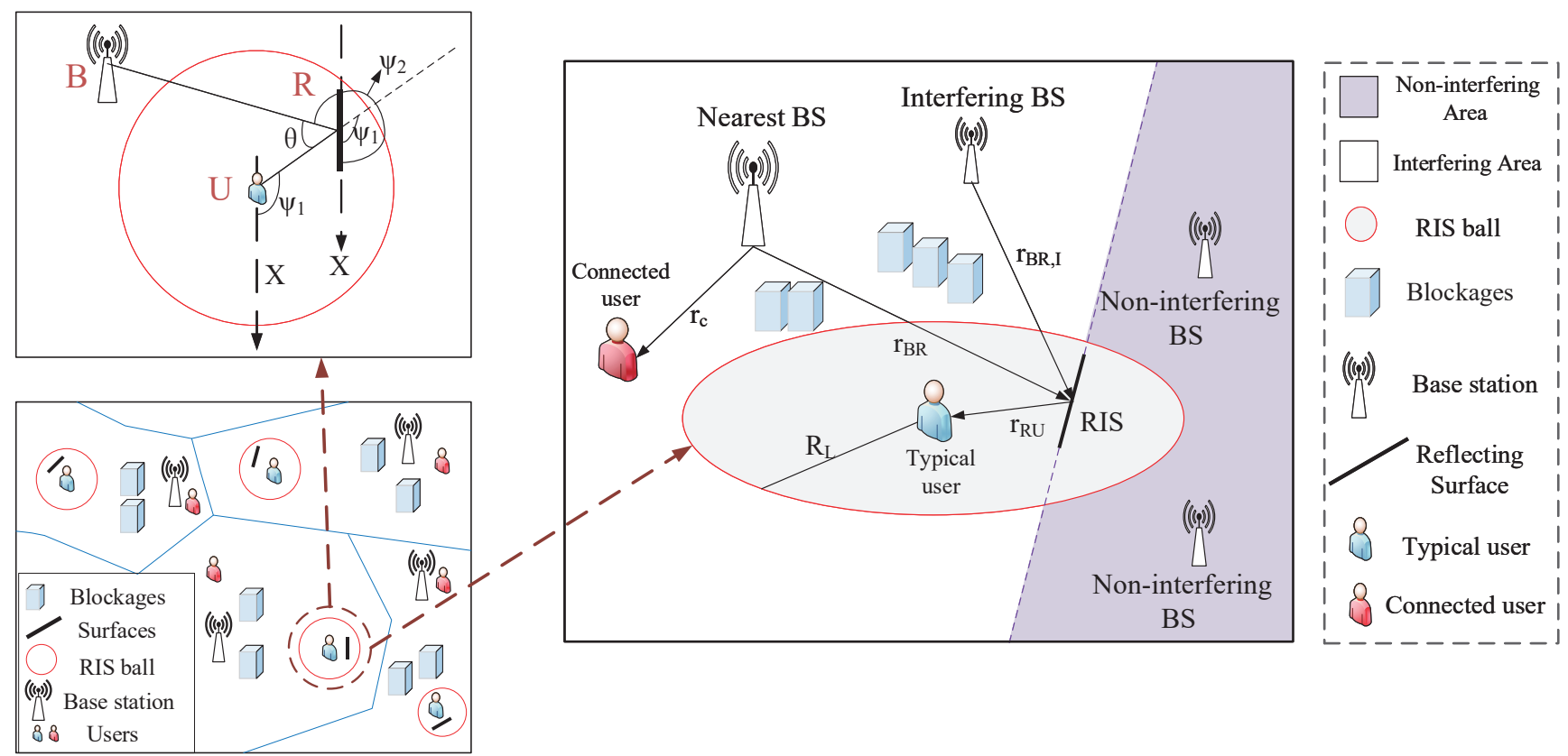

Fig. 1: Illustration of the signal model: (a) Bottom-left: An illustration of RIS-aided multi-cell scenarios; (b) Top-left: A typical NOMA network with angle demonstrations; (c) Right: A typical NOMA network with two types of BSs, such as interfering BSs facing RISs and non-interfering BSs against RISs.

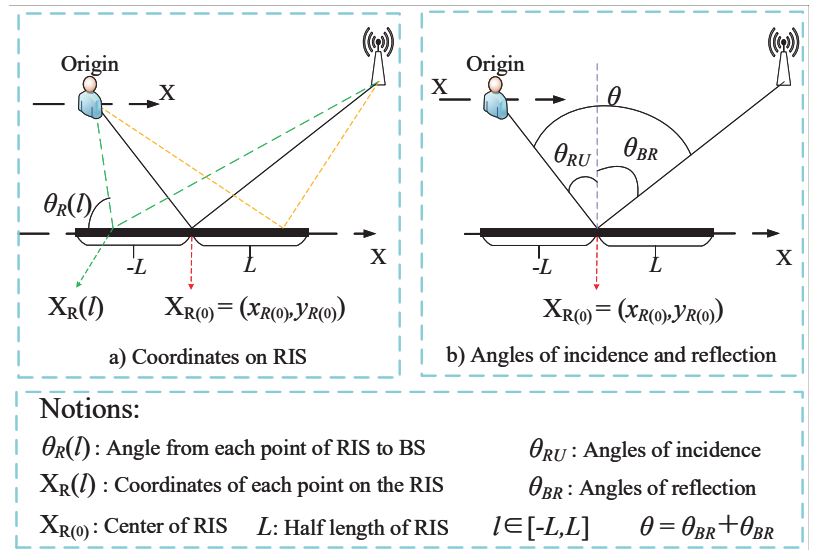

Fig. 2: Coordinates and angles in LoS balls: a) Coordinates on RIS; b) Angles of incidence and reflection

associated BS and the typical user are distributed in the same side of the RIS to establish reflecting transmission [42]. In Fig. 2, we assume the $\mathrm{x}$-axis is along the direction of RISs and the typical user is at the origin. Thus, the coordinate of each point on the RIS with a distance $l \in[-L, L]$ is expressed as $\mathbf{x}_{R}(l)=\left(x_{R}(0)+l \cos \left(\theta_{R}(l)\right), y_{R}(0)+l \sin \left(\theta_{R}(l)\right)\right) \subset \mathbb{R}^{2}$, where $\theta_{R}(l)$ is the angle to $\mathrm{x}$-axis for each point on the RIS. We set $r_{Q(0)}$ and $\theta_{Q(0)}$ as the distance and angle through the center of RISs $\mathbf{X}_{R(0)}$, where $Q \in\{B R, R U\}$ represents the links from BSs to RISs and other links from RISs to the typical user. Additionally, we assume surfaces are deployed based on long-distance communication models, thereby the surfaces receive directional lights with approximations as $r_{Q}(x) \approx r_{Q(0)}+q x \sin \left(\theta_{Q(0)}\right)$ where $q=1$ if $Q=B R$ and $q=-1$ if $Q=R U$ [7].

Influenced by devices' locations, the considered model suits for the communications with highly correlated channels, such as millimeter wave channels or $\mathrm{THz}$ channels. The channel correlation is provided in (3) and the description after (3). Hence, in Fig. 1, we exploit the physical angles, namely, angles of incident $\theta_{\mathrm{BR}}(l)$ and angles of reflection $\theta_{\mathrm{RU}}(l)$ instead of the angles of arrival (AoA) and the angles of departure (AoD). We express the AoA and AoD as $\angle A O A=\pi / 2-\theta_{\mathrm{BR}}(l)$ and $\angle A O D=\pi / 2+\theta_{\mathrm{RU}}(l)$. With the aid of AoA and $\mathrm{AoD}$, our analysis is able to provide a general guideline for designing RISs with MIMO systems. Under a high-frequency case with cylindrical electromagnetic (EM) waves [43], if one transmitting BS is located at $\mathbf{x}_{b} \in\left\{\mathbf{x}_{B}, \mathbf{x}_{I}\right\}$, the path loss model for the typical user is given by [7]

$$
\mathcal{P}_{t}\left(\mathbf{x}_{b}, \mathbf{x}_{R}, \mathbf{x}_{U}\right)=\left|\int_{-L}^{+L} \Psi(l) \exp (-j k \Omega(l)) d l\right|^{2},
$$

where

$$
\begin{aligned}
& \Psi(l)=\frac{\cos \left(\theta_{\mathrm{BR}}(l)\right)+\cos \left(\theta_{\mathrm{RU}}(l)\right)}{8 \pi \sqrt{r_{\mathrm{BR}}(l) r_{\mathrm{RU}}(l)}}, \\
& \Omega(l)=r_{\mathrm{BR}}(l)+r_{\mathrm{RU}}(l)-\Theta(l),
\end{aligned}
$$

where $k=2 \pi / \lambda$ with wavelength $\lambda$ and the communication distance for the $\mathrm{BR}$ and the RU links are $r_{\mathrm{BR}}(l)=\left\|\mathbf{x}_{b}-\mathbf{x}_{R}(l)\right\|$ and $r_{\mathrm{RU}}(l)=\left\|\mathbf{x}_{R}(l)-\mathbf{x}_{U}\right\|=$ $\| \mathbf{x}_{R}(l) \mid$, respectively, with $\theta_{\mathrm{BR}}(l)=\arcsin \left(\frac{l}{\left\|\mathbf{x}_{b}-\mathbf{x}_{R}(l)\right\|}\right)$ and $\theta_{\mathrm{RU}}(l)=\arcsin \left(\frac{l}{\left\|\mathbf{x}_{R}(l)\right\|}\right)$. Considering the reflecting point is at $\mathbf{x}_{R}(l)$, the angle of incidence $\theta_{\mathrm{BR}}(l) \in\left(0, \frac{\pi}{2}\right]$ represents the angle between the corresponding BR link and the perpendicular bisector of the RIS, whilst the angle of reflection $\theta_{\mathrm{RU}}(l) \in\left(0, \frac{\pi}{2}\right]$ is the angle between the corresponding RU link and the perpendicular bisector of the RIS. The $\Theta(l)$ is the phase-shifting parameter of RISs which is decided by the desired transmitter and receiver. 
For the connected user at $\mathbf{x}_{c} \subset \mathbb{R}^{2}$, we assume the transmission between this user and its BS follows conventional wireless communications. Therefore, the path loss expression of the connected user is as follows

$$
\mathcal{P}_{c}\left(\mathbf{x}_{b}, \mathbf{x}_{c}\right)=C\left\|\mathbf{x}_{b}-\mathbf{x}_{c}\right\|^{-\alpha_{c}},
$$

where the $C$ is intercept and $\alpha_{c}$ is the path loss exponent for the direct link. Note that the distance between the connected user and the associated BS is fixed. Therefore, $r_{c}=\left\|\mathbf{x}_{B}-\mathbf{x}_{c}\right\|$ is a constant.

\section{Signal Model}

We assume the BSs have known the demand of quality of service (QoS) of users to split delay-sensitive users and delaytolerant users, thereby the QoS-based SIC is harnessed in this paper [40]. Additionally, knowing the channel state information at BSs is able to enhance the accuracy of beamforming designs. When we consider a special case where a delaysensitive near user (the connected user) pairs with a delaytolerant far user (the typical user), the far user with SIC process may experience outage frequently because of poor channel quality. By enhancing the channel quality of the far user, RISs are exploited to improve the SIC success rate. Based on QoS-based NOMA, the associated BS allocates more transmit power to the connected user than the typical user and SIC is processed at the typical user ${ }^{5}$. Therefore, the SINR for the SIC process at the typical user is given by

$$
\gamma_{\mathrm{SIC}}=\frac{a_{c} P_{b}\left|h_{t_{1}, \mathbf{x}_{B}} h_{t_{2}, \mathbf{x}_{B}}\right|^{2} \mathcal{P}_{t}\left(\mathbf{x}_{b}, \mathbf{x}_{R}, \mathbf{x}_{U}\right)}{a_{t} P_{b}\left|h_{t_{1}, \mathbf{x}_{B}} h_{t_{2}, \mathbf{x}_{B}}\right|^{2} \mathcal{P}_{t}\left(\mathbf{x}_{b}, \mathbf{x}_{R}, \mathbf{x}_{U}\right)+I_{t, \rho_{t}}+\sigma^{2}}
$$

where

$$
I_{t, \rho_{t}}=\rho_{t} \sum_{\mathbf{x}_{I} \in \Phi_{b} \backslash \mathbf{x}_{B}} P_{b}\left|h_{t_{1}, \mathbf{x}_{I}} h_{t_{2}, \mathbf{x}_{I}}\right|^{2} \mathcal{P}_{t}\left(\mathbf{x}_{I}, \mathbf{x}_{R}, \mathbf{x}_{U}\right),
$$

and $P_{b}$ is the transmit power of BSs in each NOMA group and $\sigma^{2}$ is the variance of additive white Gaussian noise (AWGN). The $a_{t}$ and $a_{c}$ are the power allocation parameters for the typical user and the connected user, respectively. Moreover, $a_{c}>a_{t}$ and $a_{c}+a_{t}=1$. When the transmitter is at $\mathbf{x}$, for the receiver $\kappa, h_{\kappa, \mathbf{x}}$ represents its Nakagami fading term with an integer parameter $m_{\kappa}$ [44]. Additionally, $\kappa=c$ means the receiver is the connected user and $\kappa=\left\{t_{1}, t_{2}\right\}$ means the two channels from the RIS to the typical user. Additionally, we define the coefficients of Nakagami-m distributions as $m_{c}$ for the connected user and $\left\{m_{t_{1}}, m_{t_{2}}\right\}$ for the typical user. Regarding the interference $I_{t, \rho_{t}}$, since the signal from the back of RISs cannot pass through RISs, we assume $\rho_{t} \in[0,1]$ of the entire interference is able to reach the receiver $\kappa$. With the uniform deployment of BSs following the PPP, if we consider the RIS splits the area into two equal sub-areas, we have $\rho_{t}=$ 0.5 .

\footnotetext{
${ }^{5}$ In this paper, we exploit fixed power allocation coefficients. The optimized power allocation schemes will be considered in future works.
}

After the SIC process, the typical user decodes its data. The decoding SINR is expressed as

$$
\gamma_{t}=\frac{a_{t} P_{b}\left|h_{t_{1}, \mathbf{x}_{B}} h_{t_{2}, \mathbf{x}_{B}}\right|^{2} \mathcal{P}_{t}\left(\mathbf{x}_{b}, \mathbf{x}_{R}, \mathbf{x}_{U}\right)}{I_{t, \rho_{t}}+\sigma^{2}} .
$$

For the connected user, it directly decodes its messages by regarding the partner's signal as interference. Therefore, the decoding SINR for the connected user is as follows

$$
\gamma_{c}=\frac{a_{c} P_{b}\left|h_{c, \mathbf{x}_{B}}\right|^{2} \mathcal{P}_{c}\left(\mathbf{x}_{B}, \mathbf{x}_{c}\right)}{a_{t} P_{b}\left|h_{c, \mathbf{x}_{B}}\right|^{2} \mathcal{P}_{c}\left(\mathbf{x}_{B}, \mathbf{x}_{c}\right)+I_{c}+\sigma^{2}}
$$

where

$$
I_{c}=\sum_{\mathbf{x}_{I} \in \Phi_{b} \backslash \mathbf{x}_{B}} P_{b}\left|h_{c, \mathbf{x}_{I}}\right|^{2} \mathcal{P}_{c}\left(\mathbf{x}_{I}, \mathbf{x}_{c}\right) .
$$

It is worth noting that the connected user is interfered by all BSs excepting the associated BS.

\section{Channel Model Approximation}

In this section, we derive the expressions of the path loss model and angle distributions, which are fundamental for analyzing the performance of RIS-aided NOMA systems.

\section{A. Small-scale Fading Approximation}

As we consider the RIS as linear materials, we have two independent Nakagami-m fading channels for the RIS-aided link, i.e., $h_{t_{1}, \mathbf{x}_{B}}$ as the fading channel from the BS to the RIS, and $h_{t_{2}, \mathbf{x}_{B}}$ as the channel from the RIS to its aided user. Hence, the equivalent channel model is the product of these two variables, denoted as $h_{t, \mathbf{x}_{B}}=h_{t_{1}, \mathbf{x}_{B}} h_{t_{2}, \mathbf{x}_{B}}$. In power domain, the distribution of $\left|h_{t, \mathbf{x}_{B}}\right|^{2}$ is needed for the following derivations, thus we exploit the Matlab curve fitting tool to mimic the channel distribution [45]. We conclude that the distribution of $\left|h_{t, \mathbf{x}_{B}}\right|^{2}$ can be mimicked as a Gamma distribution with coefficient $m_{t}$ and $b_{t}$. This approximated distribution is presented as

$$
\begin{aligned}
f_{\left|h_{t, \mathbf{x}_{B}}\right|^{2}}(x) & =\frac{x^{m_{t}-1}}{\Gamma\left(m_{t}\right) b_{t}^{m_{t}}} \exp \left(-\frac{x}{b_{t}}\right), \\
F_{\left|h_{t, \mathbf{x}_{B}}\right|^{2}}(x) & =\frac{\gamma\left(m_{t}, x / b_{t}\right)}{\Gamma\left(m_{t}\right)},
\end{aligned}
$$

where we obtained $m_{t}=1$ and $b_{t} \approx 1$ when we set $m_{t_{1}}=$ $m_{t_{2}}=2$.

We plot the cumulative distribution function (CDF) of $\left|h_{t, \mathbf{x}_{B}}\right|^{2}$ when consider the Nakagami-m fading channel coefficient $m=[2,3,4]$ as Fig. 3(a)-3(c). Compared to the current channel models, e.g., the Meijers G-function model in [46] or the Bessel function model in [47], the advantage of our curve fitting model is that it is tractable for multi-cell scenarios as the Gamma distribution has been almost fully studied. The disadvantage is that we may be not able to find a very accurate curve fitting function, which leads to some errors. 


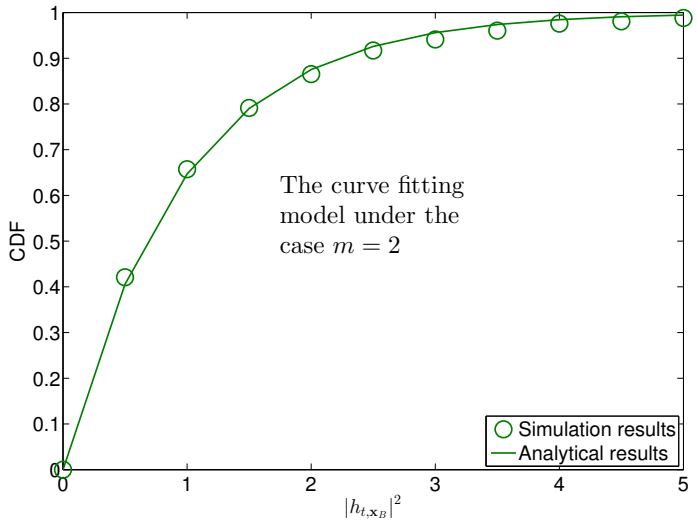

(a)



(b)

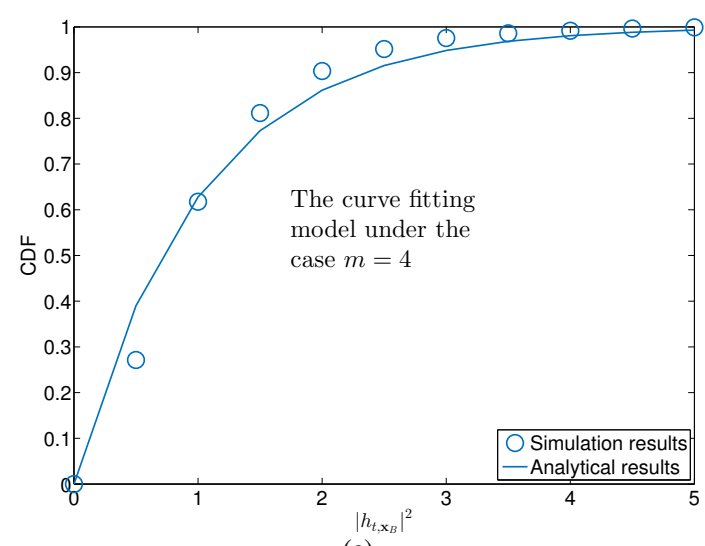

(c)

Fig. 3: The CDF of the equivalent channel model $\left|h_{t, \mathbf{x}_{B}}\right|^{2}$ with various $m$ : (a) $m=2$, (b) $m=3$, and (c) $m=4$.

\section{B. Path Loss Model}

Since surfaces are always installed on the walls of buildings, adjusting angles of surface physically may have constraints based on the shapes or directions of the walls. In this scenario, we operate the surfaces as anomalous reflectors, which is configured for reflecting waves towards a distinct direction of users. Hence, the reflection angle of waves is able to be unequal to the incidence angle.

Lemma 1. We regard surfaces as RISs rather than mirrors, thereby the angle of incidence enables to be different from the angle of reflection. As we consider the RU link as a strong LoS link, we are interested in analyzing only reflections and thus ignore parasitic scattering [12]. Under this case, we are able to obtain the phase-shifting parameter as $\Theta(l)=\left(\sin \left(\theta_{B R(0)}\right)-\sin \left(\theta_{R U(0)}\right)\right) l+\phi_{0} / k$, where $\phi_{0} \in[0,2 \pi)$ is a fixed phase shift of the direction of a user and $k=2 \pi / \lambda$ is the wavenumber with the wavelengh $\lambda$ [7]. Hence, the direction is altered to the typical user via the phase shifters. Considered on the long-distance regions with directional lights, the path loss model on RIS operations is expressed as

$$
\mathcal{P}_{t}^{R I S} \approx C_{R I S}^{2}\left(r_{B R(0)} r_{R U(0)}\right)^{-\alpha_{t}}
$$

where $C_{R I S}=\frac{L}{4 \pi}\left(\cos \left(\theta_{B R(0)}\right)+\cos \left(\theta_{R U(0)}\right)\right), \quad \alpha_{t}$ is the path loss exponent of the typical user.

Proof: Substituting $\Theta(x)$ into $\Psi(x)$, it is simplified as $\Psi(x)=r_{B R(0)}+r_{R U(0)}-\frac{\phi_{0}}{k}$. Since the BSs are assumed to emit cylindrical waves, we assume that $r_{Q}(x) \approx r_{Q(0)}+$ $q x \sin \left(\theta_{Q(0)}\right)$. Thus, the pass loss model is approximated as

$$
\begin{aligned}
\mathcal{P}_{t}^{R I S} \approx & \left|\frac{L}{4 \pi}\left(r_{B R}(l) r_{R U}(l)\right)^{-\frac{\alpha_{t}}{2}}\right|^{2} \\
& \times\left|\left(\cos \left(\theta_{B R(0)}\right)+\cos \left(\theta_{R U(0)}\right)\right)\right|^{2} \\
& \times\left|\exp \left(-j k\left(r_{B R(0)}+r_{R U(0)}-\frac{\phi_{0}}{k}\right)\right)\right|^{2},
\end{aligned}
$$

and we obtain (13) via algebraic manipulations.

\section{Distance Distributions}

Note that the users and BSs are settled via two independent HPPPs and reflecting surfaces are uniformly deployed within the ball $\mathbb{O}\left(0, R_{L}\right)$ of the typical user. Based on the MCP pattern of PCP models, locations are defined that users are parent nodes obeying HPPP and surfaces are daughter nodes within the clusters of RIS balls. Based on the aforementioned settings, we derive the probability density functions (PDFs) of distances of the corresponding cluster and other clusters for a typical user.

1) The Corresponding Cluster of the Typical User: We focus on a typical user located at the center of the RIS ball area served by uniformly distributed intelligent surfaces [48]. Thus, we are capable of deriving the PDF of the distance from a surface to its targeted typical user, denoted as $r_{R U}$, as

$$
f_{r_{R U}}(x)=\frac{2 x}{R_{L}^{2}} U\left(R_{L}-x\right)
$$

where $U(\cdot)$ is the unit step function.

2) Other Clusters of the Typical User: Since the LoS links from BSs to the typical user are blocked, we only investigate the reflecting links from the BSs to the surfaces. Thus, based on the null probability of a 2-D PPP within in the RIS ball area and order statistics $[49,50]$, the PDF of the distance between a RIS and its $n^{\text {th }}$ nearest BS is derived as

$$
f_{r_{B R}}(x, n)=\frac{2\left(\pi \lambda_{b}\right)^{n}}{(n-1) !} x^{2 n-1} \exp \left(-\pi \lambda_{b} x^{2}\right) .
$$




\section{Angle Distributions}

Shown as Fig. 1, we denote a BS as node $B$, a RIS as node $R$, and a typical user as node $U$ to clarify the angles. With the aid of a chosen positive $\mathrm{X}$-axis that is parallel to the RIS, the angles are illustrated as $\psi_{1}=\angle R U X, \psi_{2}=\angle B R X$ and $\theta=|\pi-| \psi_{2}-\psi_{1}||$. Notice that the angle of $\psi_{2}$ is uniformly distributed within $(0,2 \pi)$ based on the properties of HPPP. We additionally observe that the angle of $\psi_{1}$ obeys uniform distribution in $(0,2 \pi)$ since the RIS is uniformly distributed in the RIS ball. Based on $\psi_{1}$ and $\psi_{2}$ with the same distributions, the CDF of $\left|\psi_{2}-\psi_{1}\right|$ is derived as

$$
F_{\left|\psi_{2}-\psi_{1}\right|}(z)=\frac{4 \pi z-z^{2}}{4 \pi^{2}},
$$

therefore, the PDF of the angle of $\left|\psi_{2}-\psi_{1}\right|$ is derived as $f_{\left|\psi_{2}-\psi_{1}\right|}(z)=\frac{2 \pi-z}{2 \pi^{2}}$.

With the respect to $\theta=|\pi-| \psi_{2}-\psi_{1}||$, the CDF of the angle $\theta$ is derived as

$$
F_{\theta}(x)=F_{\left|\psi_{2}-\psi_{1}\right|}(x+\pi)-F_{\left|\psi_{2}-\psi_{1}\right|}(x-\pi)=\frac{x}{\pi},
$$

which is proved that the angle $\theta$ obeys uniform distribution within $(0, \pi)$ with the PDF as $f_{\theta}(x)=1 / \pi$.

Recall that we denote the angles of incidence as $\theta_{B R(0)}$ and the angles of reflection as $\theta_{R U(0)}$, thereby we are able to observe $\theta=\theta_{B R(0)}+\theta_{R U(0)}$ from Fig. 1. In the following, the angle analysis with respect to RISs is investigated.

When the surfaces are designed as RISs, the angles of incidence and reflection are unequal. We define the angles of incidence $\theta_{B R(0)}=\rho_{a} \theta$, where $\rho_{a} \in(0,1)$, thereby the angles of reflection is $\theta_{R U(0)}=\left(1-\rho_{a}\right) \theta$. Hence, the PDFs of the angles of incidence and reflection are derived as

$$
\begin{aligned}
& f_{\theta_{B R(0)}}(x)=\frac{1}{\rho_{a} \pi}, x \in\left(0, \frac{\pi}{2}\right), \\
& f_{\theta_{R U(0)}}(x)=\frac{1}{\left(1-\rho_{a}\right) \pi}, x \in\left(0, \frac{\pi}{2}\right) .
\end{aligned}
$$

Remark 1. As shown in Fig. 1, we note that the angles $\psi_{1}$ and $\psi_{2}$ are uniform distributed in the range of $[0,2 \pi]$. Based on the derivations in this subsection, we conclude that the BSRIS-User angle is uniformly distributed in $[0, \pi]$. Hence, we are able to extend the conclusion that any angle formed via three nodes of a PPP are uniformly distributed.

Lemma 2. Considered on an average case with a 2-D HPPP, the angles are uniformly distributed. As communication distances are independent of incidence and reflection angles, after considering the random spatial effect, the path loss of RIS links in Lemma $\mathbf{1}$ is rewritten as

$$
\mathcal{P}_{t}^{R I S}=C_{R I S, E}^{2}\left(r_{B R(0)} r_{R_{i} U(0)}\right)^{-\alpha_{t}},
$$

where $C_{R I S, E}^{2}=\frac{L^{2}}{16 \pi^{3}}\left(\pi+\frac{\sin \left(2 \rho_{a} \pi\right)}{4 \rho_{a}-12 \rho_{a}^{2}+8 \rho_{a}^{3}}\right)$.

Proof: The average path loss with respect to angles is expressed as $\mathcal{P}_{t}^{R I S}=\mathbb{E}\left[C_{R I S}^{2}\right] \times\left(r_{B R(0)} r_{R_{i} U(0)}\right)^{-\alpha_{t}}$, where $\mathbb{E}[\cdot]$ is calculating the expectation. By plugging (19) and (20), this lemma is proved.

Under a vacuum scenario with the best channel environment as mentioned in [7], the path loss exponent of reflecting links $\alpha_{t}=1$. For practical scenarios, this path loss exponent $\alpha_{t}$ should be larger than one. Therefore, we consider $\alpha_{t}>1$ in the rest of this work. The path loss exponent for conventional models are defined as $\alpha_{R F}>2$.

Remark 2. We assume the distance between the BS to the typical user as a fixed distance, denoted as $r_{f i x}$ with $r_{B R(0)}+r_{R_{i} U(0)}=\mathcal{D} r_{\text {fix }}$. We denote $P_{r}^{R I S}$ and $P_{r}^{R F}$ as the received power for RIS-aided channels and RF channels, respectively. When RF channels and RIS-aided channels follow the same kind of fading channels, we define the RIS gain as

$$
\begin{aligned}
\beta_{R I S} & =\frac{\mathbb{E}\left[P_{r}^{R I S}\right]}{\mathbb{E}\left[P_{r}^{R F}\right]}=\frac{C_{R I S, E}^{2}\left(r_{B R(0)} r_{R_{i} U(0)}\right)^{-\alpha_{t}}}{C_{R F} r_{f i x}^{-\alpha_{t}}} \\
& \geq \frac{4^{\alpha_{t}} C_{R I S, E}^{2}}{\mathcal{D}^{2} C_{R F} r_{\text {fix }}^{\alpha_{t}}}=\frac{\beta_{c} L^{2}}{r_{f i x}^{\alpha_{t}}},
\end{aligned}
$$

where $\beta_{c}=\frac{4^{\alpha_{t}}}{16 \mathcal{D}^{2} C_{R F} \pi^{3}}\left(\pi+\frac{\sin \left(2 \rho_{a} \pi\right)}{4 \rho_{a}-12 \rho_{a}^{2}+\rho_{a}^{3}}\right), \mathcal{D} \geq 1$ is an arbitrary positive number, $C_{R F}=\left(\frac{c}{4 f_{c} \pi}\right)^{2}=\frac{1}{16 \pi^{2}}\left(\frac{c}{f_{c}}\right)^{2}$ is the intercept, $c=3 \times 10^{8} \mathrm{~m} / \mathrm{s}$ is the speed of light, and $f_{c}$ is the bandwidth of users. Hence, the minimum RIS gain is directly influenced by the length of RISs and the distance between the $B S$ and the typical user. We conclude that deploying RISs at the perpendicular bisector of the BU link results in the minimum RIS gain.

Remark 3. Compared to conventional far-field propagation model $\mathcal{P}_{\text {far-field }}^{R I S}=\left(r_{B R(0)} r_{R_{i} U(0)}\right)^{-\alpha_{t}} \times C_{R F}$, the path loss model for linear materials has several differences. 1) Different path loss intercept: With $\rho_{a} \in(0,1]$, we have $C_{R I S, E}^{2}=$ $\frac{L^{2}}{16 \pi^{3}}\left(\pi+\frac{\sin \left(2 \rho_{a} \pi\right)}{4 \rho_{a}-12 \rho_{a}^{2}+8 \rho_{a}^{3}}\right) \in\left[\frac{3 L^{2}}{32 \pi^{2}}, \frac{L^{2}}{8 \pi^{2}}\right]$, which is higher than $\frac{1}{16 \pi^{2}}$ in far-field propagation model. 2) If RISs are near to the typical user, we approximately derive $\beta_{R I S} \approx\left(\frac{L f_{c}}{c}\right)^{2}$, thus we can enlarge the length of RISs to improve the path loss intercept. 3) As the channel model for linear materials is based on the Huygens-fresnel principle, it suits for both of near-field and far field scenarios with broad application scenarios.

\section{Coverage Performance Evaluation}

When we pre-decide a fixed threshold rate, the communication performance is guaranteed when the transmit rates are higher than the threshold. By defining a fixed threshold to evaluate whether the QoS of a network is satisfied, we investigate the SINR coverage performance on our RIS-aided NOMA networks based on the derived path loss expressions.

Since we exploit the typical user with SIC procedures and the messages of the connected user are directly decoded, the coverage probability expressions for the connected user and the typical use are expressed respectively as

$$
\begin{aligned}
& \mathbb{P}_{t}=\operatorname{Pr}\left\{\gamma_{\mathrm{SIC}}>\gamma_{S I C}^{t h} \mid \gamma_{t}>\gamma_{t}^{t h}\right\}, \\
& \mathbb{P}_{c}=\operatorname{Pr}\left\{\gamma_{\mathrm{c}}>\gamma_{c}^{t h}\right\}
\end{aligned}
$$

where $\operatorname{Pr}\{\cdot\}$ is the probability operation, $\gamma_{S I C}^{t h}$ is the threshold of SIC procedures, $\gamma_{t}^{t h}=2^{R_{t} / B_{w}}-1$ is the coverage threshold 
of the typical user with threshold rate $R_{t}$ and bandwidth $B_{w}$, $\gamma_{c}^{t h}=2^{R_{c} / B_{w}}-1$ is the threshold of the connected user with threshold rate $R_{c}$.

\section{A. Interference Analysis}

Before evaluating the coverage performance of this network, we would first derive the Laplace transform of interference, $I_{t, \rho_{t}}$ and $I_{c}$, under two scenarios.

1) Interference Analysis of the Connected User: Since the connected user is not served by reflecting surfaces, the Laplace transform of the interference for the connected user is expressed via conventional wireless communication analysis [48] as

$$
\mathcal{L}_{c}(s)=\mathbb{E}\left[\exp \left(-\sum_{\mathbf{x}_{I} \in \Phi_{r} \backslash \mathbf{x}_{B}} P_{b} C\left|h_{t_{1}, \mathbf{x}_{I}} h_{t_{2}, \mathbf{x}_{I}}\right|^{2} r_{c, I}-\alpha_{c}\right)\right] .
$$

Lemma 3. The Laplace transform of interference for the connected user is derived as

$$
\mathcal{L}_{c}(s)=\exp \left(-\varsigma_{1}\left({ }_{2} F_{1}\left(-\frac{2}{\alpha_{c}}, m ; 1-\frac{2}{\alpha_{c}} ;-\varsigma_{2} s\right)-1\right)\right),
$$

where ${ }_{2} F_{1}(\cdot, \cdot ; \cdot ; \cdot)$ is the hypergeometric function, $\varsigma_{1}=\pi \lambda_{b} r_{c}^{2}$ and $\varsigma_{2}=\frac{P_{b} C}{m r_{c}^{\alpha}}$.

Proof: See Appendix A.

2) Interference Analysis of the Typical User in RIS Scenari$o$ : With the aid of Lemma 2, the Laplace transform of the interference under RIS scenarios is expressed as

$$
\begin{aligned}
& \mathcal{L}_{t, R I S}(s) \\
& =\mathbb{E}\left[\exp \left(-\rho_{t} \sum_{\mathbf{x}_{I} \in \Phi_{r} \backslash \mathbf{x}_{B}} \frac{P_{b} C_{R I S, I}^{2}\left|h_{t_{1}, \mathbf{x}_{I}} h_{t_{2}, \mathbf{x}_{I}}\right|^{2}}{\left(r_{B R(I)} r_{R U(I)}\right)^{\alpha_{t}}}\right)\right] .
\end{aligned}
$$

Lemma 4. With the aid of RISs, the Laplace transform of interference for the typical user is derived as

$$
\begin{aligned}
& \mathcal{L}_{t, R I S}\left(s, r_{B R(0)}, r_{R U(0)}\right) \\
& =\exp \left(-\varsigma_{3}\left({ }_{2} F_{1}\left(-\frac{2}{\alpha_{t}}, m ; 1-\frac{2}{\alpha_{t}} ;-s \varsigma_{4}\right)-1\right)\right),
\end{aligned}
$$

where $\varsigma_{3}=\pi \lambda_{b} r_{B R(0)}^{2}$ and $\varsigma_{4}=\frac{P_{b} C_{R I S, E}^{2}}{m_{t} r_{R U(0)} r_{B R(0)}{ }^{\alpha} t}$.

Proof: See Appendix B.

\section{B. Coverage Analysis with RISs}

In this subsection, the closed-form expressions of the coverage probabilities for the typical user and the connected users are derived via Theorem 1-2 and Corollary 1-2.
1) Coverage Analysis of the Typical User with RISs: Note that the interference from the typical user is strived to be canceled with the aid of SIC procedures. When the surfaces perform as RISs, based on Lemma 2 and (23), the coverage probability is rewritten as

$$
\mathbb{P}_{t}=\operatorname{Pr}\left\{\left|h_{t, \mathbf{x}_{B}}\right|^{2}>\frac{\Upsilon\left(I_{t, \rho_{t}}+\sigma^{2}\right)}{P_{b} \mathcal{P}_{t}^{R I S}}\right\},
$$

where $\Upsilon=\max \left(\frac{\gamma_{S I C}^{t h}}{a_{c}-\gamma_{S I C}^{t h} a_{t}}, \frac{\gamma_{t}^{t h}}{a_{t}}\right)$.

Theorem 1. We assume reflecting channels as Nakagami$\mathrm{m}$ fading channels. With the aid of RISs, the approximated expression of coverage probability for the typical user is derived as

$$
\begin{aligned}
\mathbb{P}_{t} \approx & 2 \pi \lambda_{b} \int_{0}^{R_{L}} \int_{0}^{\infty} \sum_{n=1}^{m_{t}}(-1)^{n+1}\left(\begin{array}{c}
m_{t} \\
n
\end{array}\right) x \exp \left(-\beta_{0}(y) x^{\alpha_{t}}\right) \\
& \times \exp \left(-\beta_{2} x^{2}\right) d x f_{r_{R U}}(y) d y
\end{aligned}
$$

where $\quad \beta_{0}(x)=\beta_{1} x_{t}^{\alpha}, \quad \beta_{1}=\frac{n \eta_{t} \Upsilon \sigma^{2}}{P_{b} C_{R I S, E}^{2}}, \quad \beta_{2} \quad=$ $\pi \lambda_{b 2} F_{1}\left(-\frac{2}{\alpha_{t}}, m ; 1-\frac{2}{\alpha_{t}} ;-\frac{n \eta_{t} \Upsilon}{m_{t}}\right)$ and $m_{t}$ is the coefficient in Nakagami-m fading channels with unit mean values.

Proof: See Appendix $C$.

Remark 4. When we analyze the performance with respect to the length of RISs L, we simplify the expression of coverage probability based on Theorem 1 as

$$
\mathbb{P}_{t} \approx \exp \left(-\frac{A_{1}(x, y)}{L^{2}}\right) A_{2}(x, y)
$$

where $A_{1}(x, y)$ and $A_{2}(x, y)$ are other items irrelevant to the length of RISs L. Thus, we conclude that when we improve the length of RISs, the coverage performance is enhanced. This is because the large RISs are able to absorb more incident waves and reflect them to the users.

Corollary 1. Conditioned on $\alpha_{t}=2$, we are capable to derive the closed-form expression of the coverage probability for the typical user as

$$
\mathbb{P}_{t} \approx \frac{\pi \lambda_{b}}{2} \sum_{n=1}^{m_{t}}(-1)^{n+1}\left(\begin{array}{c}
m_{t} \\
n
\end{array}\right)\left(\beta_{1} R_{L}^{2}+2 \beta_{2}\right) .
$$

Proof: When $\alpha_{t}=2$, the coverage probability of the typical user is rewritten as

$$
\begin{aligned}
\mathbb{P}_{c} \approx & 2 \pi \lambda_{b} \int_{0}^{R_{L}} \int_{0}^{\infty} \sum_{n=1}^{m_{t}}(-1)^{n+1}\left(\begin{array}{c}
m_{t} \\
n
\end{array}\right) x \\
& \times \exp \left(-\left(\beta_{0}(y)+\beta_{2}\right) x^{2}\right) d x f_{r_{R U(0)}}(y) d y .
\end{aligned}
$$

With the aid of Gamma functions $\Gamma(1)=1$ and Eq. [2.3.18.2] in [51], (33) is derived as

$$
\mathbb{P}_{c} \approx \pi \lambda_{b} \sum_{n=1}^{m_{t}}(-1)^{n+1}\left(\begin{array}{c}
m_{t} \\
n
\end{array}\right) \int_{0}^{R_{L}}\left(\beta_{0}(y)+\beta_{2}\right) f_{r_{R U(0)}}(y) d y,
$$

and via several algebraic manipulations, the final expression is obtained. 
Corollary 2. Conditioned on $\alpha_{t}=4$, the closed-form expression of the coverage probability for the typical user is derived via the error function as

$$
\begin{aligned}
\mathbb{P}_{t} \approx & \sum_{n=1}^{m_{t}}(-1)^{n+1}\left(\begin{array}{c}
m_{t} \\
n
\end{array}\right) \sum_{i=1}^{K} \frac{\omega_{i} \pi^{\frac{3}{2}} \lambda_{b} \sqrt{1-\Xi_{i}^{2}}}{2 R_{L} \sqrt{\beta_{1}} \Xi_{i}} \\
& \times \exp \left(\frac{\beta_{2}^{2}}{4 \beta_{1} \Xi_{i}^{4}}\right) \operatorname{Erfc}\left(\frac{\beta_{2}}{2 \sqrt{\beta_{1}} \Xi_{\mathrm{i}}^{2}}\right),
\end{aligned}
$$

where $\eta_{t}=m_{t}\left(m_{t} !\right)^{-\frac{1}{m_{t}}}, \varpi_{i}=\cos \left(\frac{2 i-1}{2 K} \pi\right), \quad \Xi_{i}=$ $\frac{R_{L}}{2}\left(\varpi_{i}+1\right), \omega_{i}=\pi / K$ and $\operatorname{Erfc}(\cdot)$ is the error function.

Proof: Based on Appendix $C$ when $\alpha_{t}=4$, this corollary is proved via substituting (15), Eq. [2.3.15.4] in [51] and Chebyshev-gauss quadrature into (30).

2) Coverage Analysis of the Connected User: Based on (24), we are capable of rewriting the coverage probability expression as

$$
\mathbb{P}_{c}=\operatorname{Pr}\left\{\left|h_{c, \mathbf{x}_{B}}\right|^{2}<\frac{\gamma_{c}^{t h}\left(I_{c}+\sigma^{2}\right)}{\left(a_{c}-a_{t} \gamma_{c}^{t h}\right) P_{b} C r_{c}-\alpha_{c}}\right\} .
$$

Theorem 2. The closed-form expression of coverage probability for the connected users is derived as

$$
\mathbb{P}_{c} \approx \sum_{n=1}^{m_{c}}(-1)^{n+1}\left(\begin{array}{c}
m_{c} \\
n
\end{array}\right) \exp \left(-\mu_{1} r_{c}^{2}-\mu_{2} r_{c}{ }^{\alpha_{c}}\right),
$$

where $\mu_{1}=\pi \lambda_{b}\left({ }_{2} F_{1}\left(-\frac{2}{\alpha_{c}}, m ; 1-\frac{2}{\alpha_{c}} ;-\frac{n \eta_{c} \gamma_{c}^{t h}}{m\left(a_{c}-a_{t} \gamma_{c}^{t h}\right)}\right)-1\right)$, $\mu_{2}=\frac{n \eta_{c} c_{c}^{t h} \sigma^{2}}{\left(a_{c}-a_{t} \gamma_{c}^{t h}\right) P_{b} C}$ and $\eta_{c}=m_{c}\left(m_{c} !\right)^{-\frac{1}{m_{c}}}$ with Nakagami$\mathrm{m}$ fading coefficient $m_{c}$.

Proof: We exploit the Campbells theorem and Theorem 1 to derive (37).

Remark 5. When we analyze the performance with respect to the density of $B S s \lambda_{b}$, we simplify the expression of coverage probability as

$$
\mathbb{P}_{t} \approx \exp \left(-\lambda_{b} B_{1}(x, y)\right) B_{2}(x, y),
$$

where $B_{1}(x, y)$ and $B_{2}(x, y)$ are other items irrelevant to the density of $B S s \lambda_{b}$. Hence, the expression reveals that when we improve the density of $B S s \lambda_{b}$, the coverage probability is reduced. This is because the distance of connected user has been fixed and the density of BSs $\lambda_{b}$ only influence the strength of interference from other BSs.

\section{Asymptotic Coverage Probability for the Typical user}

In this subsection, we evaluate the coverage performance of the typical user when the half-length $L \rightarrow \infty$.

Corollary 3. Conditioned on $L \rightarrow \infty$, the asymptotic expression of coverage probability for the typical user is derived as

$$
\begin{aligned}
\mathbb{P}_{c} \approx & \sum_{n=1}^{m_{t}}(-1)^{n+1}\left(\begin{array}{c}
m_{t} \\
n
\end{array}\right) \frac{\pi \lambda_{b}}{\beta_{2}}-\frac{2 \pi \lambda_{b} R_{L}^{\alpha_{t}}}{\left(2+\alpha_{t}\right)} \Gamma\left(\frac{\alpha_{t}+2}{2}\right) \\
& \times \sum_{n=1}^{m_{t}}(-1)^{n+1}\left(\begin{array}{c}
m_{t} \\
n
\end{array}\right) \frac{\beta_{1}}{\beta_{2}^{\frac{\alpha_{t}+2}{2}}} .
\end{aligned}
$$

Proof: After substituting the asymptotic expression $\exp (-x)=1-x$ into the coverage probability expression, we are able to rewrite (30) as

$$
\begin{aligned}
\mathbb{P}_{c} & \approx 2 \pi \lambda_{b} \int_{0}^{R_{L}} \frac{2 y}{R_{L}^{2}} d y \int_{0}^{\infty} \sum_{n=1}^{m_{t}}\left(\begin{array}{c}
m_{t} \\
n
\end{array}\right) \frac{x \exp \left(-\beta_{2} x^{2}\right)}{(-1)^{-(n+1)}} d x \\
& -2 \pi \lambda_{b} \beta_{1} \frac{2 R_{L}^{\alpha_{t}}}{\left(2+\alpha_{t}\right)} \int_{0}^{\infty} \sum_{n=1}^{m_{t}}\left(\begin{array}{c}
m_{t} \\
n
\end{array}\right) \frac{x^{\alpha_{t}+1} \exp \left(-\beta_{2} x^{2}\right)}{(-1)^{-(n+1)}},
\end{aligned}
$$

and based on Eq. [2.3.18.2] in [51], we are able to obtain the final results.

Corollary 4. When the length of RISs are sufficiently large, we are capable to derive an upper limit from Corollary 3 as

$$
\mathbb{P}_{c} \approx \sum_{n=1}^{m_{t}}(-1)^{n+1}\left(\begin{array}{c}
m_{t} \\
n
\end{array}\right) \frac{\pi \lambda_{b}}{\beta_{2}},
$$

which can increase the calculation efficiency to a large extent.

Remark 6. Based on Huygens-Fresnel principle, this paper considers a one-dimensional (1-D) RIS [7] to evaluate the averaged performance. Considering 1-D RISs instead of twodimensional (2-D) RISs is to obtain tractable and clear derivations for further evaluation, while the properties may not be clearly reflected by exploiting the 2-D model as the complexity of derivations is high. The constraints will be released in future works.

Remark 7. This paper considers the scenarios with fixed power allocation, which is to directly present the averaged long-term performance for the whole network. We also believe that well-designed plower allocation algorithms will improve the performance of RIS-aided systems. However, this will enhance the complexity of derivations, while we still leave the space for further investigation.

\section{ERgodic Rate Evaluation}

Compared with the coverage probability with a fixed rate threshold, the achievable ergodic rate for the RIS-aided NOMA systems is opportunistically altered via the channel conditions of users. In this subsection, the closed-form expression of the ergodic rate for the typical user and the connected users are derived in the following theorems and corollaries.

\section{A. Ergodic Rate for the Typical User}

We consider the typical user to exploit the SIC procedure. The failure SIC procedure leads to the ergodic rate of the typical user always being zero. Thus, with the aid of the expression of the coverage probability, we express the ergodic rate expression of the typical user as

$$
\begin{aligned}
\mathbb{E}\left[R_{t}^{R I S}\right] & =\mathbb{E}\left[\log _{2}\left(1+\gamma_{t}\right), \gamma_{\mathrm{SIC}}>\gamma_{S I C}^{t h}\right] \\
& =\frac{1}{\ln 2} \int_{0}^{\infty} \frac{\mathbb{P}_{t}\left(\gamma_{t}^{t h}\right)}{1+\gamma_{t}^{t h}} d \gamma_{t}^{t h},
\end{aligned}
$$

and the approximated and closed-form expressions are derived in the following. 
Theorem 3. With the aid of the coverage probability expression in Theorem 1, the approximated expression of ergodic rates for the typical user is derived as

$$
\begin{aligned}
& \mathbb{E}\left[R_{t}^{R I S}\right]=\frac{2 \pi \lambda_{b}}{\ln 2 R_{L}^{2}} \int_{a_{t} \Upsilon_{1}}^{\infty} \int_{0}^{R_{L}} \int_{0}^{\infty} \sum_{n=1}^{m_{t}} \frac{2 y x(-1)^{n+1}}{1+z}\left(\begin{array}{c}
m_{t} \\
n
\end{array}\right) \\
& \times \exp \left(-\beta_{1}\left(\frac{z}{a_{t}}\right)(y x)^{\alpha_{t}}\right) \exp \left(-\beta_{2}\left(\frac{z}{a_{t}}\right) x^{2}\right) d x d y d z \\
& +\frac{2 \pi \lambda_{b}}{\ln 2 R_{L}^{2}} \int_{0}^{a_{t} \Upsilon_{1}} \int_{0}^{R_{L}} \int_{0}^{\infty} \sum_{n=1}^{m_{t}}(-1)^{n+1}\left(\begin{array}{c}
m_{c} \\
n
\end{array}\right) \\
& \times \frac{2 y x}{1+z} \exp \left(-\beta_{1}\left(\Upsilon_{1}\right)(y x)^{\alpha_{t}}\right) \exp \left(-\beta_{2}\left(\Upsilon_{1}\right) x^{2}\right) d x d y d z,
\end{aligned}
$$

where $\beta_{1}(\Upsilon(z))=\frac{n \eta_{t} \Upsilon(z) \sigma^{2}}{P_{b} C_{R I S, E}^{2}}, \Upsilon(z)=$ $\max \left(\frac{\gamma_{S I C}^{t h}}{a_{c}-\gamma_{S I C}^{t h} a_{t}}, \frac{z}{a_{t}}\right), \quad \Upsilon_{1} \quad=\quad \frac{\gamma_{S I C}^{t h}}{a_{c}-a_{t} \gamma_{S I C}^{t h}} \quad$ and $\beta_{2}(z)=\pi \lambda_{b 2} F_{1}\left(-\frac{2}{\alpha_{t}}, m ; 1-\frac{2}{\alpha_{t}} ;-\frac{n \eta_{t} \Upsilon(z)}{m_{t}}\right)$.

Proof: When considering the threshold $\gamma_{t}^{t h}$ as a variable, the condition to maintain the SIC orders as $\Upsilon(z)=$ $\max \left(\frac{\gamma_{S I C}^{t h}}{a_{c}-\gamma_{S I C}^{t h} a_{t}}, \frac{z}{a_{t}}\right)$ divides the expression into two items, such as $\gamma_{t}^{t h} \in\left[0, a_{t} \Upsilon_{1}\right]$ and $\gamma_{t}^{t h} \in\left[a_{t} \Upsilon_{1}, \infty\right]$. Thus, substituting the Laplace transform expressions of the interference caused by other RU links, this theorem is clarified.

Remark 8. As Remark 4, derivations in Theorem 3 illustrate that the ergodic rate of the typical user increases if we enhance the half-length of RISs L.

Corollary 5. When fixing the path loss exponent of the typical user $\alpha_{t}=2$, the closed-form ergodic rate expression of the typical user is derived as

$$
\begin{aligned}
& \mathbb{E}\left[R_{t}^{R I S}\right]=\frac{\pi \lambda_{b}}{2 \ln 2} \sum_{n=1}^{m_{t}}(-1)^{n+1}\left(\begin{array}{c}
m_{t} \\
n
\end{array}\right) \\
& \times\left(\sum_{j=1}^{J} \omega_{j} \sqrt{1-\Xi_{j}^{2}} \frac{a_{t} \Upsilon_{1}\left(\beta_{1}\left(\Upsilon_{1}\right) R_{L}^{2}+2 \beta_{2}\left(\Upsilon_{1}\right)\right)}{2\left(1+\Xi_{j}\right)}\right. \\
& \left.+\sum_{v=1}^{V} \omega_{v} \sqrt{1-\Xi_{v}^{2}} \frac{2 a_{t} \Upsilon_{1}\left(\beta_{1}\left(\frac{\Xi_{v}}{a_{t}}\right) R_{L}^{2}+2 \beta_{2}\left(\frac{\Xi_{v}}{a_{t}}\right)\right)}{\left(\varpi_{v}+1\right)^{2}\left(1+\Xi_{v}\right)}\right),
\end{aligned}
$$

where $\Xi_{j}=\frac{a_{t} \Upsilon_{1}}{2}\left(\varpi_{j}+1\right), \varpi_{j}=\cos \left(\frac{2 j-1}{2 J} \pi\right), \omega_{j}=\pi / J$, $\Xi_{v}=\frac{2 a_{t} \Upsilon_{1}}{\varpi_{v}+1}, \varpi_{v}=\cos \left(\frac{2 j-1}{2 V} \pi\right)$ and $\omega_{v}=\pi / V$.

Proof: Based on the closed-form expression of the coverage probability for the typical user in Corollary 1, we are able to rewrite the ergodic rate expression as

$$
\begin{aligned}
\mathbb{E} & {\left[R_{t}^{R I S}\right]=\frac{\pi \lambda_{b}}{2 \ln 2} \sum_{n=1}^{m_{t}}(-1)^{n+1}\left(\begin{array}{c}
m_{c} \\
n
\end{array}\right) } \\
& \times \int_{0}^{\infty} \frac{\beta_{1}(\Upsilon(z)) R_{L}^{2}+2 \beta_{2}(\Upsilon(z))}{1+z} d z .
\end{aligned}
$$

Based on the derivations in Theorem 3 and harnessing the Chebyshev-Gauss quadrature, we are able to derive the closedform ergodic rate expression for the typical user.
Corollary 6. When targeting the typical user's path loss exponent $\alpha_{t}=4$, we derive the closed-form ergodic rate expression of the typical user as Eq. (46) at the top of the next page, where the coefficients are the same as Corollary 5.

Proof: Substituting the closed-form coverage probability expression from Corollary 2 into (42), the ergodic rate expression is calculated as

$$
\begin{aligned}
\mathbb{E} & {\left[R_{t}^{R I S}\right]=\frac{1}{\ln 2} \sum_{n=1}^{m_{t}}(-1)^{n+1}\left(\begin{array}{c}
m_{t} \\
n
\end{array}\right) \sum_{i=1}^{K} } \\
& \times \int_{0}^{\infty} \frac{\omega_{i} \pi^{\frac{3}{2}} \lambda_{b} \sqrt{1-\Xi_{i}^{2}}}{2 R_{L} \sqrt{\beta_{1}(\Upsilon(z))} \Xi_{i}(1+z)} \\
& \times \exp \left(\frac{\beta_{2}^{2}(\Upsilon(z))}{4 \beta_{1}(\Upsilon(z)) \Xi_{i}^{4}}\right) \operatorname{Erfc}\left(\frac{\beta_{2}(\Upsilon(z))}{2 \sqrt{\beta_{1}(\Upsilon(z))} \Xi_{i}^{2}}\right) d z,
\end{aligned}
$$

and with the aid of Chebyshev-Gauss quadrature, the final expression is derived.

\section{B. Ergodic Rate for the Connected User}

Recall that the distance from the nearest BS to the connected user is fixed. We also express the expression of ergodic rate via the coverage probability expression as

$$
\mathbb{E}\left[R_{c}^{R I S}\right]=\mathbb{E}\left[\log _{2}\left(1+\gamma_{c}\right)\right]=\frac{1}{\ln 2} \int_{0}^{\infty} \frac{\mathbb{P}_{c}\left(\gamma_{c}^{t h}\right)}{1+\gamma_{c}^{t h}} d \gamma_{c}^{t h},
$$

and the approximated closed-form expression is derived via the following theorem.

Theorem 4. Since we consider the RISs to enhance the channel environments of the typical user, there is a high probability that the channel conditions of the typical user are better than the connected users. Thus, the connected user is allocated at the first stage of SIC orders to escape from the SIC procedure. In this scenario, the ergodic rate of the connected user is derived as

$$
\begin{gathered}
\mathbb{E}\left[R_{c}^{R I S}\right]=\frac{1}{\ln 2} \sum_{n=1}^{m_{c}}(-1)^{n+1}\left(\begin{array}{c}
m_{c} \\
n
\end{array}\right) \sum_{w=1}^{W} \omega_{w} \sqrt{1-\Xi_{w}^{2}} \\
\times \frac{\Upsilon_{2}}{2\left(1+\Xi_{w}\right)} \exp \left(-\mu_{1}\left(\Xi_{w}\right) r_{c}^{2}-\mu_{2}\left(\Xi_{w}\right) r_{c}{ }^{\alpha_{c}}\right),
\end{gathered}
$$

where $\Upsilon_{2}=\frac{a_{c}}{a_{t}}, \Xi_{w}=\frac{\Upsilon_{2}}{2}\left(\varpi_{w}+1\right), \varpi_{w}=\cos \left(\frac{2 w-1}{2 W} \pi\right)$ and $\omega_{w}=\pi / W$.

Proof: Based on the conditions when deriving the coverage probability, we obtain that $\gamma_{c}^{t h}<\frac{a_{c}}{a_{t}}$. Therefore, the range of $\gamma_{c}^{\text {th }}$ is in $\left[0, \Upsilon_{2}\right]$. Based on the coverage probability expression of the connected user, we express the ergodic rate expression as

$$
\begin{aligned}
& \mathbb{E}\left[R_{c}^{R I S}\right]=\frac{1}{\ln 2} \sum_{n=1}^{m_{c}}(-1)^{n+1}\left(\begin{array}{c}
m_{c} \\
n
\end{array}\right) \\
& \quad \times \int_{0}^{\Upsilon_{2}} \frac{1}{1+z} \exp \left(-\mu_{1}(z) r_{c}^{2}-\mu_{2}(z) r_{c}{ }^{\alpha_{c}}\right) d z,
\end{aligned}
$$




$$
\begin{aligned}
& \mathbb{E}\left[R_{t}^{R I S}\right]=\sum_{n=1}^{m_{t}} \frac{(-1)^{n+1}}{\ln 2}\left(\begin{array}{c}
m_{t} \\
n
\end{array}\right) \sum_{i=1}^{K} \sum_{j=1}^{J} \omega_{i} \omega_{j} \frac{a_{t} \Upsilon_{1} \pi^{\frac{3}{2}} \lambda_{b} \sqrt{1-\Xi_{i}^{2}} \sqrt{1-\Xi_{j}^{2}}}{4 R_{L} \sqrt{\beta_{1}\left(\Upsilon_{1}\right)} \Xi_{i}(1+z)} \exp \left(\frac{\beta_{2}^{2}\left(\Upsilon_{1}\right)}{4 \beta_{1}\left(\Upsilon_{1}\right) \Xi_{i}^{4}}\right) \operatorname{Erfc}\left(\frac{\beta_{2}\left(\Upsilon_{1}\right)}{2 \sqrt{\beta_{1}\left(\Upsilon_{1}\right)} \Xi_{i}^{2}}\right) \\
& \quad+\sum_{n=1}^{m_{t}} \frac{(-1)^{n+1}}{\ln 2}\left(\begin{array}{c}
m_{c} \\
n
\end{array}\right) \sum_{i=1}^{K} \sum_{v=1}^{V} \omega_{i} \omega_{v} \frac{a_{t} \Upsilon_{1} \pi^{\frac{3}{2}} \lambda_{b} \sqrt{1-\Xi_{i}^{2}} \sqrt{1-\Xi_{v}^{2}}}{R_{L} \sqrt{\beta_{1}\left(\frac{\Xi_{v}}{a_{t}}\right)} \Xi_{i}\left(1+\Xi_{v}\right)^{3}} \exp \left(\frac{\beta_{2}^{2}\left(\frac{\Xi_{v}}{a_{t}}\right)}{4 \beta_{1}\left(\frac{\Xi_{v}}{a_{t}}\right) \Xi_{i}^{4}}\right) \operatorname{Erfc}\left(\frac{\beta_{2}\left(\frac{\Xi_{v}}{a_{t}}\right)}{2 \sqrt{\beta_{1}\left(\frac{\Xi_{v}}{a_{t}}\right)} \Xi_{i}^{2}}\right), \quad(46)
\end{aligned}
$$

and utilizing Chebyshev-Gauss quadrature, the proof is accomplished.

Remark 9. Based on the analytical procedure in Remark 5, we are able to conclude that the ergodic rate of the connected user increases when the density of BSs $\lambda_{b}$ is enhanced.

\section{Asymptotic Ergodic Rate for the Typical User}

In this subsection, we evaluate the coverage performance of the typical user when $L \rightarrow \infty$ holds. With the aid of the asymptotic expression of the exponential functions, we derive the asymptotic coverage probability and diversity gains.

Corollary 7. We assume the half-length of the RIS to infinity, denoted as $L \rightarrow \infty$. With the aid of asymptotic expression, such as $\exp (-x)=1-x$ when $x \rightarrow 0$, we derive the approximated expression of the ergodic rate as

$$
\begin{aligned}
\mathrm{E} & {\left.\left[R_{t}^{R I S}\right]\right|_{L \rightarrow \infty} } \\
= & \frac{2 \pi \lambda_{b}}{\ln 2 R_{L}^{2}} \int_{0}^{\infty} \int_{0}^{R_{L}} \int_{0}^{\infty} \sum_{n=1}^{m_{t}}(-1)^{n+1}\left(\begin{array}{c}
m_{t} \\
n
\end{array}\right) \frac{2 y x}{1+z} \\
& \times\left(1-\beta_{1}(\Upsilon(z))(y x)^{\alpha_{t}}\right) \exp \left(-\beta_{2}(\Upsilon(z)) x^{2}\right) d x d y d z \\
= & C_{1}-C_{2} f\left(\frac{1}{L^{2}}\right)
\end{aligned}
$$

where $C_{1}$ and $C_{2}$ are constants and $f\left(\frac{1}{L^{2}}\right)$ represents a function negatively correlated to $L^{2}$.

Proof: Substituting the asymptotic expression into the expression in Theorem 3, we achieve the asymptotic expression.

Remark 10. When the condition $L \rightarrow \infty$ holds, which means we have a large RIS surface, we derive the slope to evaluate the performance as

$$
S=\lim _{L \rightarrow \infty} \frac{\left.\mathrm{E}\left[R_{t}^{R I S}\right]\right|_{L \rightarrow \infty}}{\log (L)}=0 .
$$

Remark 11. Since the slope versus $L$ is zero, which represents that when we enhance the length of RISs, the performance would increase to an upper limit eventually shown as Fig. 4. This is because enhancing $L$ is directly increases the path loss gain. Although the path loss gain of the typical user would increase when we continuously enlarge the RISs, the interference from other BSs would also be enhanced, which leads to an upper limit 6 .

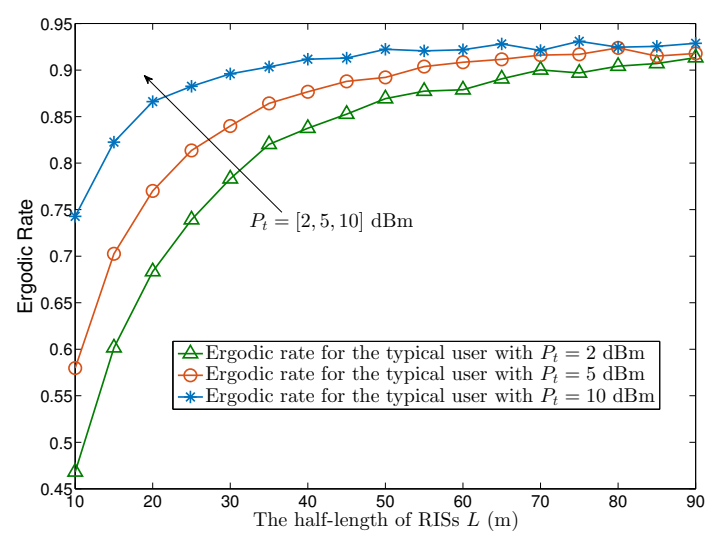

Fig. 4: The ergodic rate versus the half-length of the RIS $L$.

\section{NUMERICAL RESULtS}

In this section, numerical results are indicated to validate analytical and asymptotic expressions of coverage probability derived in the previous sections. We further accomplish the simulation results of the ergodic rate performance, including analytical and asymptotic expressions. Several comparisons are proposed to compare the performance under several cases, such as RIS-aided NOMA, RIS-aided OMA, and non-RISaided NOMA.

\section{A. Simulation Results on Coverage Probability}

In this subsection, numerical results validate analytical coverage probability for the typical user (Theorem 1) and the connected user (Theorem 2). Additionally, the asymptotic expressions via the length of RISs are validated to match the simulation results in high length region. Without otherwise specification, we define the numerical settings as shown in the Table I.

1) Validation of Results on Coverage Probability: The analytical coverage probability expressions of the typical user (Theorem 1) and the connected user (Theorem 2) are validated in Fig. 5(a). Additionally, we compare the performance of several scenarios with different density of BSs $\lambda_{b}$ in Fig. 5(a).

${ }^{6} \mathrm{We}$ note that the path loss gain is in influenced by the distance between the BSs to the typical user as well, resulting in the path loss gain of the interference is lower than that of the associated BS. However, if we keep increasing the length of the RISs, there will be a point that the strength of the interference catches up to the strength of the signals, which causes an upper limit. 
TABLE I: Network Parameters

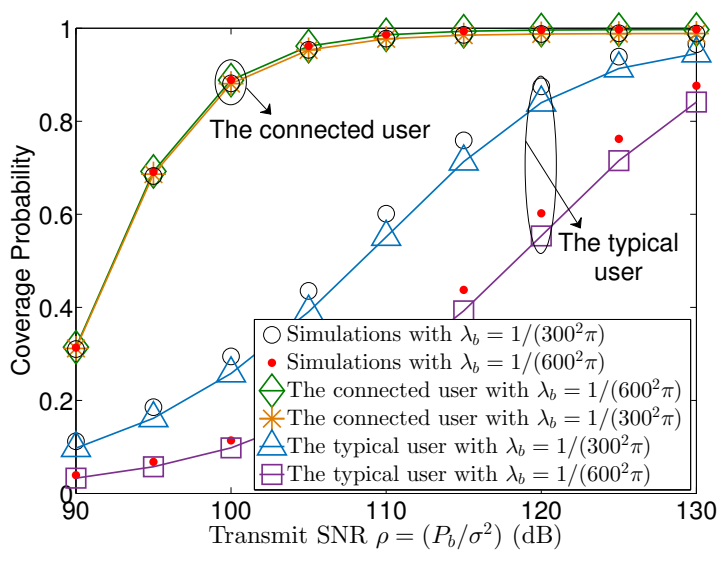

(a)

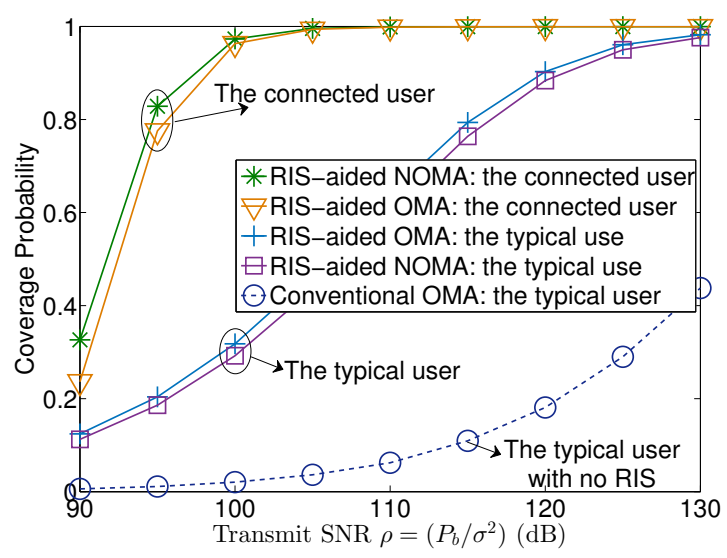

(b)



(c)

Fig. 5: (a) Coverage probability versus transmit SNR with various density of BSs $\lambda_{b}=\left[1 /\left(300^{2} \pi\right), 1 /\left(600^{2} \pi\right)\right]$ : a verification of Theorem 1 and Theorem 2. (b) Coverage probability versus transmit SNR: a comparison among conventional OMA, RIS-aided OMA and RIS-aided NOMA scenarios. (c) Coverage probability versus transmit SNR with various halflength of RISs $L=[2,3,4] \mathrm{m}$ and path loss exponents $\alpha_{t}=[2.5,3,4]$.

\begin{tabular}{|l|l|}
\hline The length of RIS $L$ & $4 \mathrm{~m}$ \\
\hline The noise power & $\begin{array}{l}\sigma^{2}=-170+10 \log \left(f_{c}\right)+ \\
N_{f}=-90 \mathrm{~dB}\end{array}$ \\
\hline The bandwidth of the carrier $f_{c}$ & $10 \mathrm{MHz}$ \\
\hline $\begin{array}{l}\text { The noise figure from hardware } \\
N_{f}\end{array}$ & $10 \mathrm{~dB}$ \\
\hline The transmit power of users $P_{t}$ & {$[0,40] \mathrm{dBm}$} \\
\hline $\begin{array}{l}\text { The path loss exponents } \alpha_{c} \text { and } \\
\alpha_{t}\end{array}$ & 3.3 \\
\hline The RIS ball radius $R_{L}$ & $20 \mathrm{~m}$ \\
\hline The density of BSs $\lambda_{b}$ & $\lambda_{b}=1 /\left(300^{2} \pi\right)$ \\
\hline The coverage and SIC thresholds & $\gamma_{S I C}^{t h}=\gamma_{t}^{t h}=\gamma_{c}^{t h}=10^{-2}$ \\
\hline $\begin{array}{l}\text { The Gamma distribution coeffi- } \\
\text { cients }\end{array}$ & $m_{c}=m_{t_{1}}=m_{t_{2}}=2$ \\
\hline The power allocation coefficients & $a_{c}=0.6$ and $a_{t}=0.4$ \\
\hline $\begin{array}{l}\text { The distance between the con- } \\
\text { nected user and BS }\end{array}$ & $r_{C}=200 \mathrm{~m}$ \\
\hline
\end{tabular}

The observation is that the typical user is more sensitive than the connected user on coverage performance when the density of BSs is changed. The reason is clarified in the following. Based on the QoS-based SIC, the connected user considers the typical user's signals as interference. As the power level of the typical user's signals is higher than that of the interference from interfering BSs, the influence of enhancing the density of interfering BSs for the connected user is limited. For the typical user, the interference from interfering BSs is enhanced by the RIS and the connected user's signals are removed by SIC. Thus, the density of BSs directly influences the strength of interference for the typical user.

2) Performance Comparison: The performance among conventional OMA, RIS-aided OMA, and RIS-aided OMA scenarios is compared in Fig. 5(b), which demonstrates that the performance of NOMA users boosts considerably with the aid of RISs, especially for the typical user. The enhancement of NOMA users are able to be explained that 1) when assisted with RISs, the connected users enable to avoid SIC procedures since the typical user with substantially improved channel gains takes over the SIC procedures, thereby the connected user would not experience outage scenarios caused by SIC failures; 2) with the aid of RISs, superior channel gains of the typical user increase coverage performance.

3) Outage Performance on Path Loss Exponents: Considering the length of RISs, Fig. 5(c) investigates the performance varied by $L$ and path loss exponents. Two observations are apparent to explain that: 1) long lengths of RISs cause high performance since more reconfigurable meta-material elements are involved and 2) enlarging path loss exponents results in reduced performance as the relationship between the path loss exponents and the coverage performance is a negative correlation.

4) Outage Performance on the Length of RIS: In Fig. 6(a), we investigate the outage performance versus the length of RIS. One observation is obtained that the coverage probability is enhanced when the length of RISs increases. This is because that larger RISs integrate more energy with the typical user. When we enlarge the radius of the RIS serving area, the performance of the typical user is reduced as the averaged 




(a)

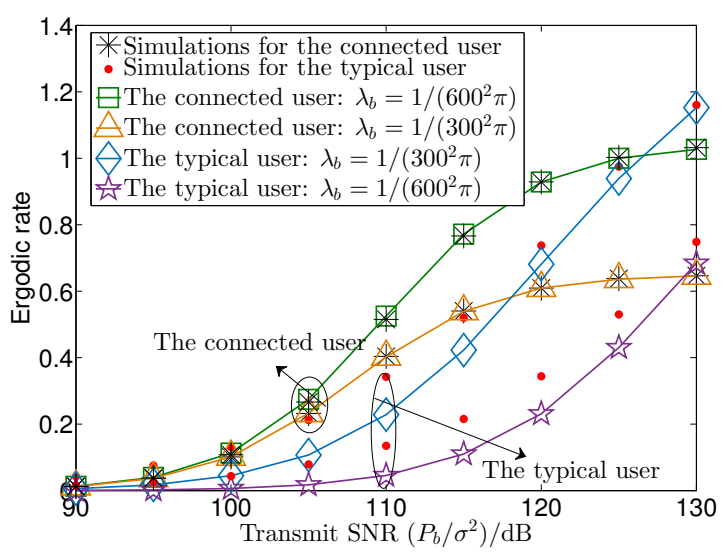

(b)



(c)

Fig. 6: (a) Coverage probability versus the half-length of RISs $L \mathrm{~m}$ with various radii of RIS serving area as $R_{L}=[10,15,30] \mathrm{m}$. (b) Ergodic rates (BPCU) versus transmit SNR with various density values of BSs $\lambda_{b}=$ $\left[1 /\left(200^{2} \pi\right), 1 /\left(400^{2} \pi\right), 1 /\left(600^{2} \pi\right)\right]$ for the typical user. (c) Ergodic rates (BPCU) versus transmit SNR with various distances between the connected user and the BS as $r_{C}=$ $[50,75,100] \mathrm{m}$ for the connected user. distance from the typical user to its associated BS is enlarged, which leads to large path loss.

\section{B. Simulation Results on Ergodic Rage}

In this subsection, we validate analytical ergodic rate via numerical results for the typical user (Theorem 3) and connected users (Theorem 4). We note that the unit of ergodic rate is the bit per cell use (BPCU). We define the numerical settings as the same as the sections on coverage probability without otherwise specification.

1) Validation of Ergodic Rate for the Typical User: The ergodic rate performance with the unit BPCU versus the transmit SNR $P_{b} / \sigma^{2}$ is indicated in Fig. 6(b). We observe that when we have dense BSs, the performance of the typical user outperforms than users with small density. This is because a large density of BSs contributes to a short averaged distance between the nearest BS and the typical user, which leads to better performance than small density situations.

2) Validation of Ergodic Rate for the connected User: We validated the analytical results of the ergodic rate for the connected user via Fig. 6(c), which demonstrates the ergodic rate (BPCU) versus the transmit SNR. Observed by this figure, we conclude that when we increase the distance between the connected user and the nearest BS, the performance goes worse as the long-distance leads to heavy path loss.

\section{CONCLUSION}

This paper has investigated the coverage probability and ergodic rate of RIS-assisted NOMA frameworks, where the PCP principle has been invoked to capture the spatial effects of NOMA users. The path loss models of RIS reflecting links has been derived, which is correlated with the "product of distances" model to conform with long-distance regions. The angle distributions have been presented with a conclusion that the BS-RIS-User angles obey uniform distributions in $[0 . \pi]$. With the aid of the derived closed-form expressions of coverage probabilities and numerical results, the performance of conventional OMA, RIS-aided NOMA, and RIS-aided OMA scenarios has been compared, which has shown that RISs enhance the performance of users significantly. The asymptotic expressions of ergodic rates for the typical user have illustrated that the performance has upper limits when enhancing the length of the RIS. The analysis of this paper has verified that two applications of RISs in multi-cell NOMA networks are feasible, such that: 1) RISs are able to improve the channel conditions of blocked or far users; 2) RISs enable to alter the SIC order to maintain primary users avoiding SIC procedures. For future works, we will consider more practical scenarios, i.e., the optimal power allocation methods, two dimensional RISs, other stochastic geometry models. Additionally, we will evaluate the performance of a user aided by more than two RISs.

\section{Appendix A: Proof of Lemma 3}

With the aid of the expansion of the exponential function and based on (25), we are able to express the Laplace 
transform of the interference for the connected user as

$$
\begin{aligned}
& \mathcal{L}_{c}(s)=\mathbb{E}\left[\exp \left(-\sum_{\mathbf{x}_{I} \in \Phi_{r} \backslash \mathbf{x}_{B}} s P_{b} C\left|h_{c, \mathbf{x}_{I}}\right|^{2} r_{c, I}{ }^{-\alpha_{c}}\right)\right] \\
& =\mathbb{E}\left[\prod_{\mathbf{x}_{I} \in \Phi_{r} \backslash \mathbf{x}_{B}} \exp \left(-s P_{b} C\left|h_{c, \mathbf{x}_{I}}\right|^{2} r_{c, I}{ }^{-\alpha_{c}}\right)\right] .
\end{aligned}
$$

Based on the binomial expansion, we obtain a tight upper bound for the normalized Gamma variables $|h|^{2}$, denoted as $\mathbb{P}\left\{|h|^{2}<x\right\}=(1-\exp (-x \eta))^{m}$, where $m$ is the coefficient of Gamma distribution and $\eta=m(m !)^{-\frac{1}{m}}$. Thus, the equation (A.1) is approximately expressed as

$$
\mathcal{L}_{c}(s)=\mathbb{E}\left[\prod_{\mathbf{x}_{I} \in \Phi_{r} \backslash \mathbf{x}_{B}}\left(1+\frac{s P_{b} C r_{c, I}{ }^{-\alpha_{c}}}{m}\right)^{-m}\right] .
$$

For some stochastic processes, the probability generating functional (PGFL) are useful tools for dealing with sums and limits of random variables, which is expressed as

$$
\mathbb{E}\left[\prod_{x \in \Phi} f(x)\right]=\exp \left(-\lambda \int_{\mathbb{R}^{2}}(1-f(x)) d x\right)
$$

where $\Phi$ is a PPP with density $\lambda$ and $f(x): \mathbb{R}^{2} \rightarrow[0,1]$ is a real value function. Then, we utilize PGFL to derive the Laplace transform as

$L_{c}(s)=\exp \left(-2 \pi \lambda_{b} \int_{r_{c}}^{\infty}\left(1-\left(1+\frac{s P_{b} C r^{-\alpha_{c}}}{m}\right)^{-m}\right) r d r\right)$,

and via the integration $\int_{A}^{\infty}\left(1-\left(1+s y^{-\alpha}\right)^{-N}\right) y d y=\frac{A^{2}}{2} \times$ $\left({ }_{2} F_{1}\left(-\frac{2}{\alpha}, N ; 1-\frac{2}{\alpha} ;-\frac{s}{A^{\alpha}}\right)-1\right)$, this lemma is proved.

\section{APPENDIX B: PROOF OF LEMMA 4}

Based on the properties of exponential functions such as $e^{a+b}=e^{a} e^{b}$, the Laplace transform expressions of the interference from other cells for the typical user is expressed as

$$
\begin{gathered}
\mathcal{L}_{t, R I S}(s)=\mathbb{E}\left[\exp \left(\sum_{\substack{\mathbf{x}_{I} \in \Phi_{r} \\
\mathbf{x}_{I} \neq \mathbf{x}_{B}}} \frac{-\rho_{t} s P_{b}\left|h_{t_{1}, \mathbf{x}_{I}} h_{t_{2}, \mathbf{x}_{I}}\right|^{-2}}{C_{R I S I}^{-2}\left(r_{B R(I)} r_{R U(I)}\right)^{\alpha_{t}}}\right)\right] \\
=\mathbb{E}\left[\prod_{\mathbf{x}_{I} \in \Phi_{r} \backslash \mathbf{x}_{B}}\left(1+\frac{s P_{b} C_{R I S, I}^{2}}{2 m\left(r_{B R(I)} r_{R U(I)}\right)^{\alpha_{t}}}\right)^{-m}\right] \cdot
\end{gathered}
$$

Based on the PGFL theorem, the Laplace transform in (B.1) is derived as

$$
\begin{aligned}
& \mathcal{L}_{t, R I S}(s)= \\
& \exp \left(-2 \pi \lambda_{b} \int_{r_{B R(0)}}^{\infty}\left(1-\left(1+\frac{s P_{b} C_{R I S, E}^{2}}{m\left(x r_{R U(0)}\right)^{\alpha_{t}}}\right)^{-m}\right) x d x\right) .
\end{aligned}
$$

Exploiting the integration expressions in Appendix A, the lemma is proved.

\section{Appendix C: Proof of Theorem 1}

The Campbell's theorem is expressed as $\mathbb{E}\left[\sum_{x \in \Phi} f(x)\right]=$ $\lambda \int_{\mathbb{R}^{2}} f(x) d x$, where $\Phi$ is a PPP with density $\lambda$. Based on the Campbell's theorem, the expressions of the average interference for the connected user is derived as

$$
\mathbb{E}\left[I_{c}\right]=2 \pi \lambda_{b} \int_{r_{c}}^{\infty} P_{b} C r^{1-\alpha_{c}} d r=\frac{2 \pi \lambda_{b} P_{b} C r_{c}^{2-\alpha_{c}}}{\alpha_{c}-2} .
$$

Note that the normalized Gamma variables have a tight upper bound, denoted as $\mathbb{P}\left[\left|h^{2}\right|<x\right]<\left(1-e^{-x \eta_{t}}\right)^{m_{t}}$. Utilizing binomial expansions, the expressions of coverage probability for the typical user is expressed as

$$
\mathbb{P}_{t} \approx \sum_{n=1}^{m_{t}}(-1)^{n+1}\left(\begin{array}{c}
m_{t} \\
n
\end{array}\right) \mathcal{L}_{t, R I S}\left[\frac{n \eta_{t} \Upsilon I_{t, \rho_{t}}}{P_{b} \mathcal{P}_{t}^{R I S}}\right] \mathbb{E}\left[e^{-\frac{n \eta_{t} \Upsilon \sigma^{2}}{P_{b} \mathcal{P}_{t}^{R I S}}}\right],
$$

and via substituting Lemma 2 and Lemma 4 into the equation above, the theorem is verified.

\section{REFERENCES}

[1] C. Zhang, W. Yi, Y. Liu, Z. Qin, and K. K. Chai, "Downlink analysis for reconfigurable intelligent surfaces aided NOMA networks," Proc. IEEE Global Commun. Conf. (GLOBECOM), pp. 1-6, 2020.

[2] Y. Liu, Z. Qin, M. Elkashlan, Z. Ding, A. Nallanathan, and L. Hanzo, "Nonorthogonal multiple access for 5G and beyond," Proc. IEEE, vol. 105 , no. 12 , pp. 2347-2381, 2017.

[3] M. A. ElMossallamy, H. Zhang, L. Song, K. G. Seddik, Z. Han, and G. Y. Li, "Reconfigurable intelligent surfaces for wireless communications: Principles, challenges, and opportunities," IEEE Trans. Cogn. Commun. Netw., vol. 6, no. 3, pp. 990-1002, 2020.

[4] Q. Wu and R. Zhang, "Towards smart and reconfigurable environment: Intelligent reflecting surface aided wireless network," IEEE Commun. Mag., vol. 58, no. 1, pp. 106-112, Jan. 2020.

[5] Y. Liu, Z. Ding, M. Elkashlan, and H. V. Poor, "Cooperative nonorthogonal multiple access with simultaneous wireless information and power transfer," IEEE J. Sel. Areas Commun., vol. 34, no. 4, pp. 938953, Apr. 2016.

[6] W. Tang, M. Z. Chen, X. Chen, J. Y. Dai, Y. Han, M. Di Renzo, Y. Zeng, S. Jin, Q. Cheng, and T. J. Cui, "Wireless communications with reconfigurable intelligent surface: Path loss modeling and experimental measurement," IEEE Trans. Wireless Commun., vol. 20, no. 1, pp. 421439, 2021.

[7] M. Di Renzo, F. Habibi Danufane, X. Xi, J. de Rosny, and S. Tretyakov, "Analytical modeling of the path-loss for reconfigurable intelligent surfaces-anomalous mirror or scatterer?" Proc. IEEE 21th Int. Workshop Signal Process. Adv. Wireless Commun. (SPAWC), pp. 1-5, 2020.

[8] E. Basar, M. Di Renzo, J. De Rosny, M. Debbah, M. Alouini, and R. Zhang, "Wireless communications through reconfigurable intelligent surfaces," IEEE Access, vol. 7, pp. 116753-116773, 2019.

[9] G. Pan, J. Ye, J. An, and M. S. Alouini, "When full-duplex transmission meets intelligent reflecting surface: Opportunities and challenges," arXiv preprint arXiv:2005.12561, 2019.

[10] Y. Liu, Z. Qin, M. Elkashlan, A. Nallanathan, and J. A. McCann, "Nonorthogonal multiple access in large-scale heterogeneous networks," IEEE J. Sel. Areas Commun., vol. 35, no. 12, pp. 2667-2680, Dec. 2017.

[11] W. Yi, Y. Liu, E. Bodanese, A. Nallanathan, and G. K. Karagiannidis, "A unified spatial framework for UAV-aided mmWave networks," IEEE Trans. Commun., vol. 67, no. 12, pp. 8801-8817, 2019.

[12] T. Bai and R. W. Heath, "Coverage and rate analysis for millimeter-wave cellular networks," IEEE Trans. Wireless Commun., vol. 14, no. 2, pp. $1100-1114,2015$

[13] W. Yan, X. Yuan, Z. Q. He, and X. Kuai, "Passive beamforming and information transfer design for reconfigurable intelligent surfaces aided multiuser MIMO systems," IEEE J. Sel. Areas Commun., vol. 38, no. 8, pp. 1793-1808, 2020 . 
[14] M. Jung, W. Saad, M. Debbah, and C. S. Hong, "On the optimality of reconfigurable intelligent surfaces (RISs): Passive beamforming, modulation, and resource allocation," IEEE Trans. Wireless Commun., Early access, 2021.

[15] B. Di, H. Zhang, L. Song, Y. Li, Z. Han, and H. V. Poor, "Hybrid beamforming for reconfigurable intelligent surface based multi-user communications: Achievable rates with limited discrete phase shifts," IEEE J. Sel. Areas Commun., vol. 38, no. 8, pp. 1809-1822, 2020.

[16] J. Ye, S. Guo, and M. Alouini, "Joint reflecting and precoding designs for SER minimization in reconfigurable intelligent surfaces assisted MIMO systems," IEEE Trans. Wireless Commun., vol. 19, no. 8, pp. 5561-5574, 2020.

[17] H. Guo, Y. Liang, J. Chen, and E. G. Larsson, "Weighted sumrate maximization for reconfigurable intelligent surface aided wireless networks," IEEE Trans. Wireless Commun., vol. 19, no. 5, pp. 3064 3076, 2020.

[18] T. Hou, Y. Liu, Z. Song, X. Sun, and Y. Chen, "MIMO-NOMA networks relying on reconfigurable intelligent surface: A signal cancellation-based design," IEEE Trans. Commun., vol. 68, no. 11, pp. 6932-6944, 2020.

[19] C. Huang, R. Mo, and C. Yuen, "Reconfigurable intelligent surface assisted multiuser MISO systems exploiting deep reinforcement learning," IEEE J. Sel. Areas Commun., vol. 38, no. 8, pp. 1839-1850, 2020.

[20] S. Khan and S. Y. Shin, "Deep-learning-aided detection for reconfigurable intelligent surfaces," arXiv preprint arXiv:1910.09136, 2019.

[21] X. Yang, C. K. Wen, and S. Jin, "MIMO detection for reconfigurable intelligent surface-assisted millimeter wave systems," IEEE J. Sel. Areas Commun., vol. 38, no. 8, pp. 1777-1792, 2020.

[22] N. S. Perović, M. Di Renzo, and M. F. Flanagan, "Channel capacity optimization using reconfigurable intelligent surfaces in indoor mmWave environments," Proc. IEEE Int. Commun. Conf. (ICC), pp. 1-7, 2020.

[23] A. U. Makarfi, K. M. Rabie, O. Kaiwartya, O. S. Badarneh, X. Li, and R. Kharel, "Reconfigurable intelligent surface enabled IoT networks in generalized fading channels," Proc. IEEE Int. Commun. Conf. (ICC), pp. 1-6, 2020.

[24] A. Khaleel and E. Basar, "Reconfigurable intelligent surface-empowered MIMO systems," IEEE Syst. J., pp. 1-9, 2020.

[25] M. Zeng, X. Li, G. Li, W. Hao, and O. A. Dobre, "Sum rate maximization for IRS-assisted uplink NOMA," IEEE Commun. Lett., vol. 25, no. 1, pp. 234-238, 2021.

[26] V. C. Thirumavalavan and T. S. Jayaraman, "BER analysis of reconfigurable intelligent surface assisted downlink power domain NOMA system," Proc. Int. Conf. Commun. Syst. Netw. (COMSNETS), pp. 519522, 2020.

[27] J. Zhu, Y. Huang, J. Wang, K. Navaie, and Z. Ding, "Power efficient IRS-assisted NOMA," IEEE Trans. Commun., vol. 69, no. 2, pp. 900913, 2021.

[28] B. Zheng, Q. Wu, and R. Zhang, "Intelligent reflecting surface-assisted multiple access with user pairing: NOMA or OMA?" IEEE Commun. Lett., vol. 24, no. 4, pp. 753-757, 2020.

[29] M. Fu, Y. Zhou, Y. Shi, and K. B. Letaief, "Reconfigurable intelligent surface empowered downlink non-orthogonal multiple access," arXiv preprint arXiv:1910.07361, 2019.

[30] Z. Ding and H. Vincent Poor, "A simple design of IRS-NOMA transmission," IEEE Commun. Lett., vol. 24, no. 5, pp. 1119-1123, 2020.

[31] Z. Ding, R. Schober, and H. V. Poor, "On the impact of phase shifting designs on IRS-NOMA," IEEE Wireless Commun. Lett., vol. 9, no. 10, pp. 1596-1600, 2020.

[32] T. Hou, Y. Liu, Z. Song, X. Sun, Y. Chen, and L. Hanzo, "Reconfigurable intelligent surface aided NOMA networks," IEEE J. Sel. Areas Commun., vol. 38, no. 11, pp. 2575-2588, 2020.

[33] Y. Cheng, K. H. Li, Y. Liu, K. C. Teh, and H. Vincent Poor, "Downlink and uplink intelligent reflecting surface aided networks: NOMA and OMA," IEEE Trans. Wireless Commun., pp. 1-1, 2021.

[34] T. Hou, Y. Liu, Z. Song, X. Sun, Y. Chen, and L. Hanzo, "MIMO assisted networks relying on large intelligent surfaces: A stochastic geometry model," arXiv preprint arXiv:1910.00959, 2019.

[35] W. Mei and R. Zhang, "Performance analysis and user association optimization for wireless network aided by multiple intelligent reflecting surfaces," IEEE Trans. Commun., vol. 69, no. 9, pp. 6296-6312, Sep. 2021.

[36] H. Xie, J. Xu, and Y.-F. Liu, "Max-min fairness in IRS-aided multi-cell MISO systems with joint transmit and reflective beamforming," IEEE Trans. Wireless Commun., vol. 20, no. 2, pp. 1379-1393, Feb 2021.

[37] Z. Zhang and L. Dai, "A joint precoding framework for wideband reconfigurable intelligent surface-aided cell-free network," IEEE Trans. Signal Process., vol. 69, pp. 4085-4101, 2021.

[38] C. Pan, H. Ren, K. Wang, W. Xu, M. Elkashlan, A. Nallanathan, and L. Hanzo, "Multicell MIMO communications relying on intelligent reflecting surfaces," IEEE Trans. Wireless Commun., vol. 19, no. 8, pp. 5218-5233, Aug. 2020.

[39] W. Ni, X. Liu, Y. Liu, H. Tian, and Y. Chen, "Resource allocation for multi-cell IRS-aided NOMA networks," IEEE Trans. Wireless Commun., vol. 20, no. 7, pp. 4253-4268, Jul. 2021.

[40] Z. Ding, R. Schober, and H. V. Poor, "Unveiling the importance of SIC in NOMA systems-part 1: State of the art and recent findings," IEEE Commun. Lett., vol. 24, no. 11, pp. 2373-2377, Nov. 2020.

[41] W. Yi, Y. Liu, and A. Nallanathan, "Modeling and analysis of D2D millimeter-wave networks with poisson cluster processes," IEEE Trans. Commun., vol. 65, no. 12, pp. 5574-5588, Dec 2017.

[42] M. Di Renzo and J. Song, "Reflection probability in wireless networks with metasurface-coated environmental objects: an approach based on random spatial processes," EURASIP J. on Wireless Commun. and Netw., vol. 2019, no. 1, p. 99, 2019.

[43] M. Di Renzo, K. Ntontin, J. Song, F. H. Danufane, X. Qian, F. Lazarakis, J. De Rosny, D. Phan-Huy, O. Simeone, R. Zhang, M. Debbah, G. Lerosey, M. Fink, S. Tretyakov, and S. Shamai, "Reconfigurable intelligent surfaces vs. relaying: Differences, similarities, and performance comparison," IEEE Open J. Commun. Soc., vol. 1, pp. 798-807, 2020.

[44] W. Yi, Y. Liu, Y. Deng, A. Nallanathan, and R. W. Heath, "Modeling and analysis of mmWave V2X networks with vehicular platoon systems," IEEE J. Sel. Areas Commun., vol. 37, no. 12, pp. 2851-2866, 2019.

[45] C. Zhang, W. Yi, Y. Liu, Z. Ding, and L. Song, "STAR-IOS aided NOMA networks: Channel model approximation and performance analysis," arXiv preprint arXiv:2107.01543, 2021.

[46] H. Ibrahim, H. Tabassum, and U. T. Nguyen, "Exact coverage analysis of intelligent reflecting surfaces with Nakagami-m channels," IEEE Trans. Veh. Technol., vol. 70, no. 1, pp. 1072-1076, Jan. 2021.

[47] D. Selimis, K. P. Peppas, G. C. Alexandropoulos, and F. I. Lazarakis, "On the performance analysis of RIS-empowered communications over Nakagami-m fading," IEEE Commun. Lett., vol. 25, no. 7, pp. 21912195, Jul. 2021.

[48] Y. Liu, Z. Qin, M. Elkashlan, Y. Gao, and L. Hanzo, "Enhancing the physical layer security of non-orthogonal multiple access in large-scale networks," IEEE Trans. Wireless Commun., vol. 16, no. 3, pp. 1656$1672,2017$.

[49] D. Moltchanov, "Distance distributions in random networks," Ad Hoc Networks, vol. 10, no. 6, pp. 1146-1166, 2012.

[50] J. G. Andrews, F. Baccelli, and R. K. Ganti, "A tractable approach to coverage and rate in cellular networks," IEEE Trans. Commun., vol. 59, no. 11, pp. 3122-3134, 2011.

[51] A. P. Prudnikov, Y. A. Brychkov, and O. I. Marichev, "Integrals and series, vol. 1, special functions," 1986.

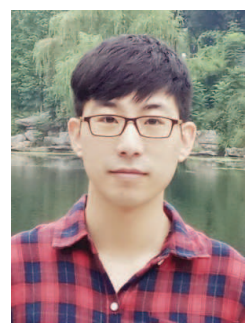

networks.
Chao Zhang (Graduate Student Member, IEEE) received the B.S. degree in Electrical Engineering from the Southwest University, China, in 2018. He is currently pursuing the Ph.D. degree with the Communication Systems Research Group, School of Electronic Engineering and Computer Science, Queen Mary University of London.

His research interests are on performance analysis including grant-free transmission, stochastic geometry, non-orthogonal multiple access techniques (NOMA), and reconfigurable intelligent surface (RIS) 


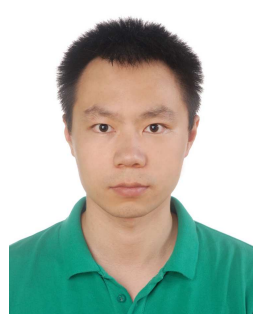

Wenqiang Yi (Member, IEEE) received the Ph.D. degree in electrical engineering from Queen Mary University of London, U.K., in 2020. He is currently a Post-Doctoral Researcher with Communication Systems Research Group, School of Electronic Engineering and Computer Science, Queen Mary University of London, since 2020.

His research interests include next generation multiple access (NGMA), millimetre-wave communications, stochastic geometry, and reinforcement learning. He received Chinese Government Award for Self-Financed Outstanding Students Abroad, 2020. He received the Exemplary Reviewer of the IEEE COMMUNICATION LETTERS and the IEEE TRANSACTIONS ON COMMUNICATIONS in 2019 and 2020. He has served as a TPC Member for many IEEE conferences, such as GLOBECOM, VTC, etc. He serves as the Secretary of the Special Interest Group on Signal Processing Techniques for NGMA by the Signal Processing and Computing for Communications Technical Committee.

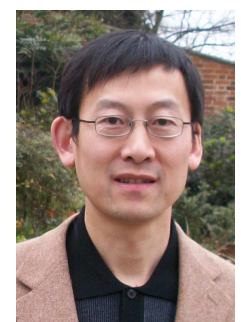

Kun Yang (Senior Member, IEEE) received his $\mathrm{PhD}$ from the Department of Electronic and Electrical Engineering of University College London (UCL), UK. He is currently a Chair Professor in the School of Computer Science and Electronic Engineering, University of Essex, leading the Network Convergence Laboratory (NCL), UK. He is also an affiliated professor at UESTC, China. Before joining in the University of Essex at 2003, he worked at UCL on several European Union (EU) research projects for several years. His main research interests include wireless networks and communications, IoT networking, data and energy integrated networks and mobile computing. He manages research projects funded by various sources such as UK EPSRC, EU FP7/H2020 and industries. He has published $200+$ journal papers and filed 20 patents. He serves on the editorial boards of both IEEE (e.g., IEEE TNSE, IEEE ComMag, IEEE WCL) and non-IEEE journals. He is a Member of Academia Europaea (MAE). He is a Senior Member of IEEE (since 2008), a Fellow of IET and a Fellow of BCS. He is an IEEE ComSoc Distinguished Lecturer (2020-2021).

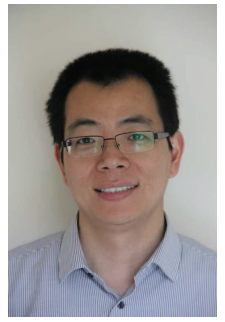

Zhiguo Ding (Fellow, IEEE) received his B.Eng in Electrical Engineering from the Beijing University of Posts and Telecommunications in 2000, and the $\mathrm{Ph} . \mathrm{D}$ degree in Electrical Engineering from Imperial College London in 2005. From Jul. 2005 to Apr. 2018, he was working in Queen's University Belfast, Imperial College, Newcastle University and Lancaster University. Since Apr. 2018, he has been with the University of Manchester as a Professor in Communications. From Oct. 2012 to Sept. 2021, he has also been an academic visitor in Princeton University. Dr. Ding's research interests are 5G networks, game theory, cooperative and energy harvesting networks and statistical signal processing. He is serving as an Area Editor for the IEEE Open Journal of the Communications Society, an Editor for IEEE Transactions on Vehicular Technology, and Journal of Wireless Communications and Mobile Computing, and was an Editor for IEEE Wireless Communication Letters, IEEE Transactions on Communications, IEEE Communication Letters from 2013 to 2016. He recently received the EU Marie Curie Fellowship 2012-2014, the Top IEEE TVT Editor 2017, IEEE Heinrich Hertz Award 2018, IEEE Jack Neubauer Memorial Award 2018, IEEE Best Signal Processing Letter Award 2018, and Friedrich Wilhelm Bessel Research Award 2020. He is a Fellow of the IEEE, a Distinguished Lecturer of IEEE ComSoc, and a Web of Science Highly Cited Researcher in two categories 2020 . 